\title{
Nonlinear Dynamical Stability of Newtonian Rotating White Dwarfs and Supermassive Stars
}

\author{
Tao Luo \& Joel Smoller
}

\begin{abstract}
We prove general nonlinear stability and existence theorems for rotating star solutions which are axi-symmetric steady-state solutions of the compressible isentropic EulerPoisson equations in 3 spatial dimensions. We apply our results to rotating and nonrotating white dwarf, and rotating high density supermassive (extreme relativistic) stars, stars which are in convective equilibrium and have uniform chemical composition. This paper is a continuation of our earlier work ([28]).
\end{abstract}

\section{Contents}

1 Introduction $\quad 1$

2 Rotating Star Solutions

3 General Existence and Stability Theorems 6

3.1 Compactness of Minimizing Sequence . . . . . . . . . . . . . . 7

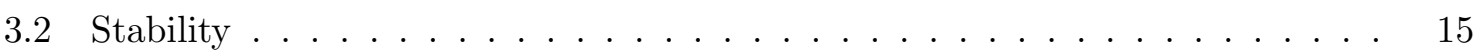

4 Applications to White Dwarf and Supermassive Stars $\quad 23$

5 Nonlinear Dynamical Stability of Non-Rotating White Dwarf Stars With General Perturbations $\quad 27$

\section{Introduction}

The motion of a compressible isentropic perfect fluid with self-gravitation is modeled by the Euler-Poisson equations in three space dimensions (cf [6]):

$$
\left\{\begin{array}{l}
\rho_{t}+\nabla \cdot(\rho \mathbf{v})=0 \\
(\rho \mathbf{v})_{t}+\nabla \cdot(\rho \mathbf{v} \otimes \mathbf{v})+\nabla p(\rho)=-\rho \nabla \Phi \\
\Delta \Phi=4 \pi \rho .
\end{array}\right.
$$


Here $\rho, \mathbf{v}=\left(v_{1}, v_{2}, v_{3}\right), p(\rho)$ and $\Phi$ denote the density, velocity, pressure and gravitational potential, respectively. The gravitational potential is given by

$$
\Phi(x)=-\int_{\mathbb{R}^{3}} \frac{\rho(y)}{|x-y|} d y=-\rho * \frac{1}{|x|},
$$

where $*$ denotes convolution. System (1.1) is used to model the evolution of a Newtonian gaseous star ([6]). In the study of time-independent solutions of system (1.1), there are two cases, non-rotating stars and rotating stars. An important question concerns the stability of such solutions. Physicists call such star solutions stable provided that they are minima of an associated energy functional ([37], p.305 \& [33]). Mathematicians, on the other hand, consider dynamical nonlinear stability via solutions of the Cauchy problem. The main purpose of this paper is to prove a general theorem which relates these two notions and shows that for a wide class of Newtonian rotating stars, minima of the energy functional are in fact, dynamically stable. This is done for various equations of state $p=p(\rho)$ which includes polytropes, supermassive, and white dwarf stars.

For non-rotating stars, Rein ([32]) has proved nonlinear stability under various hypotheses on the equation of state, including in particular, polytropes where $p=k \rho^{\gamma}, \gamma>4 / 3$; his theory applies to neither white dwarf nor supermassive stars. In a recent paper, [28], we studied nonlinear stability of rotating polytropic stars, where $p=k \rho^{\gamma}, \gamma>4 / 3$. In this paper, we generalize these results to rotating white dwarf and supermassive stars, thereby completing the nonlinear stability theory for rotating (and non-rotating) compressible Newtonian stars*.

Our main theorem applies to minimizers of an energy functional with a total mass constraint. The crucial hypotheses are that the infimum of the energy functional in the requisite class, be finite and negative. This is verified for both white dwarf and supermassive stars by combining a scaling technique used by Rein ([31]), together with our method in [28] where we use some particular solutions of the Euler-Poisson equations in order to simplify the energy functional. It should be noticed that neither the scaling technique in [31] nor the method in 28] using particular solutions of Euler-Poisson equations apply to white dwarf stars directly. As a bi-product of our method, we prove the existence of a minimizer for the energy functional, which is a rotating white dwarf star solution, in a class of functions having less symmetry than those solutions obtained in [2] and [13]. The method in [2] and [13] is to construct a specific minimizing sequence of the energy functional, each element in the sequence being a steady solution of the Euler-Poisson equations. In contrast, our method is to show that any minimizing sequence of the energy functional must be compact (cf. Theorem 3.1 below). This fact is crucial for both existence and stability results.

\footnotetext{
* In all cases under consideration, stability is only "conditional" because no global in time solutions have been constructed so far for compressible Euler-type equations in three spatial dimensions; this is a major open problem.
} 
For a white dwarf star (a star in which gravity is balanced by electron degeneracy pressure), the pressure function $p(\rho)$ obeys the following asymptotics ([ $]$, Chapter 10):

$$
\left\{\begin{array}{l}
p(\rho)=c_{1} \rho^{4 / 3}-c_{2} \rho^{2 / 3}+\cdots, \quad \rho \rightarrow \infty \\
p(\rho)=d_{1} \rho^{5 / 3}-d_{2} \rho^{7 / 3}+O\left(\rho^{3}\right), \quad \rho \rightarrow 0
\end{array}\right.
$$

where $c_{1}, c_{2}, d_{1}$ and $d_{2}$ are positive constants. The existence theory for non-rotating white dwarf stars is classical provided the mass $M$ of the star is not greater than a critical mass $M_{c}\left(M \leq M_{c}\right)([\underline{6})$. For rotating white dwarf stars with prescribed total mass and angular momentum distribution, Auchumuty and Beals ([2]) proved that if the angular momentum distribution is nonnegative, then existence holds if $M \leq M_{c}$. Friedman and Turkington ([13]) proved existence for any mass provided that the angular momentum distribution is everywhere positive; see Li ([23]), Chanillo \& Li ([7]) and Luo \& Smoller ([27]) for related results for rotating star solutions with prescribed constant angular velocity. To the best of our knowledge, our stability theorem in this paper for rotating and non-rotating white dwarf stars with $M \leq M_{c}$ is the first nonlinear dynamical stability theorem for such stars.

For a supermassive star (a star which is supported by the pressure of radiation rather than that of matter; sometimes called an extreme relativistic degenerate star [33]), the pressure $p(\rho)$ is given by $([37])$ :

$$
p(\rho)=k \rho^{\gamma}, \gamma=4 / 3
$$

where $k>0$ is a constant. For non-rotating spherically symmetric solutions for supermassive stars, Weinberg ([37]) showed that the total energy vanishes; thus to quote Weinberg ([37], p. 327) "the polytrope with $\gamma=4 / 3$ is trembling between stability and instability", and he remarks that one needs to use general relativity to settle this stability problem. For rotating supermassive star solutions, we show here that the energy is negative $E<0$ due to the rotational kinetic energy (see (4.26) below). Thus the stability problem falls within the framework of Newtonian mechanics and so our general stability theorem applies to show that rotating supermassive stars are nonlinearly stable, provided that $M \leq M_{c}$.

For the stability of both white dwarfs and supermassive stars, we require that the total mass of each one lies below to a corresponding critical mass, a "Chandrasekhar" limit. We show that this holds because the pressure function for both is of the order $\rho^{4 / 3}$ as $\rho \rightarrow \infty$.

The above dynamical stability results for rotating stars apply for axi-symmetric perturbations. For non-rotating stars, G. Rein ([32]) proved nonlinear dynamical stability for general perturbations. However, his result does not apply to white dwarf stars. For non-rotating white dwarf stars, the problem was formulated by Chandrasekhar [5] in 1931 (and also in [11] and [19]) and leads to an equation for the density which was called the "Chandrasekhar equation" by Lieb and Yau in 24]. This equation predicts the gravitational collapse at some critical mass ([5] and [6]). This gravitational collapse was also verified by Lieb and Yau ([24]) as the limit of Quantum Mechanics. In Section 5, we prove the nonlinear dynamical stability 
for non-rotating white dwarf stars with general perturbations provided that the total mass is below some critical mass.

Other related results besides those mentioned above for compressible fluid rotating stars can be found in [3], 4], [12], and [27].

The linearized stability and instability for non-rotating and rotating stars were discussed by Lin ([25] ), Lebovitz ([21]) and Lebovitz \& Lifschitz ([22]). Related nonlinear stability and instability results for gaseous stellar objects can be found in Guo \& Rein ([15], [16]) and Jang ([14]). Related results for the Euler-Poisson equations of self-gravitating fluids can be found in [10], 18], 29] and [36].

\section{Rotating Star Solutions}

We now introduce some notation which will be used throughout this paper. We use $\int$ to denote $\int_{\mathbb{R}^{3}}$, and use $\|\cdot\|_{q}$ to denote $\|\cdot\|_{L^{q}\left(\mathbb{R}^{3}\right)}$. For any point $x=\left(x_{1}, x_{2}, x_{3}\right) \in \mathbb{R}^{3}$, let

$$
r(x)=\sqrt{x_{1}^{2}+x_{2}^{2}}, z(x)=x_{3}, B_{R}(x)=\left\{y \in \mathbb{R}^{3},|y-x|<R\right\} .
$$

For any function $f \in L^{1}\left(\mathbb{R}^{3}\right)$, we define the operator $B$ by

$$
B f(x)=\int \frac{f(y)}{|x-y|} d y=f * \frac{1}{|x|} .
$$

Also, we use $\nabla$ to denote the spatial gradient, i.e., $\nabla=\nabla_{x}=\left(\partial_{x_{1}}, \partial_{x_{2}}, \partial_{x_{3}}\right)$. $C$ will denote a generic positive constant.

A rotating star solution $(\tilde{\rho}, \tilde{\mathbf{v}}, \tilde{\Phi})(r, z)$, where $r=\sqrt{x_{1}^{2}+x_{2}^{2}}$ and $z=x_{3}, x=\left(x_{1}, x_{2}, x_{3}\right) \in$ $\mathbb{R}^{3}$, is an axi-symmetric time-independent solution of system (1.1), which models a star rotating about the $x_{3}$-axis. Suppose the angular momentum (per unit mass), $J\left(m_{\tilde{\rho}}(r)\right)$ is prescribed, where

$$
m_{\tilde{\rho}}(r)=\int_{\sqrt{x_{1}^{2}+x_{2}^{2}}<r} \tilde{\rho}(x) d x=\int_{0}^{r} 2 \pi s \int_{-\infty}^{+\infty} \tilde{\rho}(s, z) d s d z
$$

is the mass in the cylinder $\left\{x=\left(x_{1}, x_{2}, x_{3}\right): \sqrt{x_{1}^{2}+x_{2}^{2}}<r\right\}$, and $J$ is a given function. In this case, the velocity field $\tilde{\mathbf{v}}(x)=\left(v_{1}, v_{2}, v_{3}\right)$ takes the form

$$
\tilde{\mathbf{v}}(x)=\left(-\frac{x_{2} J\left(m_{\tilde{\rho}}(r)\right)}{r^{2}}, \frac{x_{1} J\left(m_{\tilde{\rho}}(r)\right)}{r^{2}}, 0\right) .
$$

Substituting this in (1.1), we find that $\tilde{\rho}(r, z)$ satisfies the following two equations:

$$
\left\{\begin{array}{l}
\partial_{r} p(\tilde{\rho})=\tilde{\rho} \partial_{r}(B \tilde{\rho})+\tilde{\rho} L\left(m_{\tilde{\rho}}(r) r^{-3}\right. \\
\partial_{z} p(\tilde{\rho})=\tilde{\rho} \partial_{z}(B \tilde{\rho})
\end{array}\right.
$$


where the operator $B$ is defined in (2.2), and

$$
L\left(m_{\tilde{\rho}}\right)=J^{2}\left(m_{\tilde{\rho}}\right)
$$

is the square of the angular momentum. We define

$$
A(\rho)=\rho \int_{0}^{\rho} \frac{p(s)}{s^{2}} d s .
$$

It is easy to verify that (cf. [2]) (2.4) is equivalent to

$$
A^{\prime}(\tilde{\rho}(x))+\int_{r(x)}^{\infty} L\left(m_{\tilde{\rho}}(s) s^{-3} d s-B \tilde{\rho}(x)=\lambda, \quad \text { where } \tilde{\rho}(x)>0,\right.
$$

for some constant $\lambda$. Here $r(x)$ and $z(x)$ are as in (2.1). Let $M$ be a positive constant and let $W_{M}$ be the set of functions $\rho$ defined by ,

$$
\begin{aligned}
W_{M}= & \left\{\rho: \mathbb{R}^{3} \rightarrow \mathbb{R}, \rho \text { is axisymmetric, } \rho \geq 0, \text { a.e. },\right. \\
& \left.\int \rho(x) d x=M, \int\left(A(\rho(x))+\frac{\rho(x) L\left(m_{\rho}(r(x))\right)}{r(x)^{2}}+\rho(x) B \rho(x)\right) d x<+\infty .\right\}
\end{aligned}
$$

For $\rho \in W_{M}$, we define the energy functional $F$ by

$$
F(\rho)=\int\left[A(\rho(x))+\frac{1}{2} \frac{\rho(x) L\left(m_{\rho}(r(x))\right)}{r(x)^{2}}-\frac{1}{2} \rho(x) B \rho(x)\right] d x .
$$

In (2.7), the first term denotes the potential energy, the middle term denotes the rotational kinetic energy and the third term is the gravitational energy.

For a white dwarf star, the pressure function $p(\rho)$ satisfies the following conditions:

$$
\lim _{\rho \rightarrow 0+} \frac{p(\rho)}{\rho^{4 / 3}}=0, \lim _{\rho \rightarrow \infty} \frac{p(\rho)}{\rho^{4 / 3}}=\mathfrak{K}, p^{\prime}(\rho)>0 \text { as } \rho>0,
$$

where $\mathfrak{K}$ is a finite positive constant. Assuming that the function $L \in C^{1}[0, M]$ and satisfies

$$
L(0)=0, L(m) \geq 0, \text { for } 0 \leq m \leq M,
$$

Auchmuty and Beals (cf. [2]) proved the existence of a minimizer of the functional $F(\rho)$ in the class of functions $W_{M, S}=W_{M} \cap W_{S}$, where

$$
W_{S}=\left\{\rho: \mathbb{R}^{3} \rightarrow \mathbb{R}, \rho\left(x_{1}, x_{2},-x_{3}\right)=\rho\left(x_{1}, x_{2}, x_{3}\right), x_{i} \in \mathbb{R}, i=1,2,3\right\} .
$$

Their result is given in the following theorem.

Theorem 2.1. ([2]). If the pressure function p satisfies (2.8) (for either $0<\mathfrak{K}<+\infty$ or $\mathfrak{K}=+\infty$ and (2.9) holds, then there exists a constant $M_{c}>0$ depending on the constant $\mathfrak{K}$ in (2.8) (if $\mathfrak{K}=+\infty$ then $M_{c}=+\infty$, if $0<\mathfrak{K}<+\infty$, then $0<M_{c}<+\infty$ ) such that, if

$$
M<M_{c}
$$


then there exists a function $\hat{\rho}(x) \in W_{M, S}$ which minimizes $F(\rho)$ in $W_{M, S}$. Moreover, if

$$
G=\left\{x \in \mathbb{R}^{3}: \hat{\rho}(x)>0\right\},
$$

then $\bar{G}$ is a compact set in $\mathbb{R}^{3}$, and $\hat{\rho} \in C^{1}(G) \cap C^{\beta}\left(\mathbb{R}^{3}\right)$ for some $0<\beta<1$. Furthermore, there exists a constant $\mu<0$ such that

$$
\left\{\begin{array}{l}
A^{\prime}(\hat{\rho}(x))+\int_{r(x)}^{\infty} L\left(m_{\hat{\rho}}(s) s^{-3} d s-B \hat{\rho}(x)=\mu, \quad x \in G,\right. \\
\int_{r(x)}^{\infty} L\left(m_{\hat{\rho}}(s) s^{-3} d s-B \hat{\rho}(x) \geq \mu, \quad x \in \mathbb{R}^{3}-G .\right.
\end{array}\right.
$$

Remark 1. When $0<\mathfrak{K}<\infty$, the constant $0<M_{c}<+\infty$ in (2.11) is called critical mass. The critical mass was first found by Chandrasekhar (cf. [6]) in the study of non-rotating white dwarf stars. When $0<\mathfrak{K}<\infty$, it was proved by Friedman and Turkington ([13]) that, if the angular momentum satisfies the following condition

$$
J \in C^{1}([0, M]), J^{\prime}(m) \geq 0, \text { for } 0 \leq m \leq M, J(0)=0, J(m)>0 \text { for } 0<m \leq M,
$$

where $J$ is the angular momentum, then the condition (2.11) can be removed, i.e., the above theorem holds for any positive total mass $M$.

In this paper, we are interested in the minimizer of functional $F$ in the larger class $W_{M}$. By the same argument as in [2], it is easy to prove the following theorem on the regularity of the minimizer.

Theorem 2.2. Suppose that the pressure function $p$ satisfies:

$$
\lim _{\rho \rightarrow 0+} \frac{p(\rho)}{\rho^{6 / 5}}=0, \lim _{\rho \rightarrow \infty} \frac{p(\rho)}{\rho^{6 / 5}}=\infty, p^{\prime}(\rho)>0 \text { as } \rho>0,
$$

and the angular momentum satisfies (2.9). Let $\tilde{\rho}$ be a minimizer of the energy functional $F$ in $W_{M}$ and let

$$
\Gamma=\left\{x \in \mathbb{R}^{3}: \tilde{\rho}(x)>0\right\},
$$

then $\tilde{\rho} \in C\left(\mathbb{R}^{3}\right) \cap C^{1}(\Gamma)$. Moreover, there exists a constant $\lambda$ such that

$$
\left\{\begin{array}{l}
A^{\prime}(\tilde{\rho}(x))+\int_{r(x)}^{\infty} L\left(m_{\tilde{\rho}}(s) s^{-3} d s-B \tilde{\rho}(x)=\lambda, \quad x \in \Gamma,\right. \\
\int_{r(x)}^{\infty} L\left(m_{\tilde{\rho}}(s) s^{-3} d s-B \tilde{\rho}(x) \geq \lambda, \quad x \in \mathbb{R}^{3}-\Gamma .\right.
\end{array}\right.
$$

We call such a minimizer $\tilde{\rho}$ a rotating star solution with total mass $M$ and angular momentum $\sqrt{L(m)}$.

\section{General Existence and Stability Theorems}

For the angular momentum, besides the condition (2.9), we also assume that it satisfies the following conditions:

$$
L(a m) \geq a^{4 / 3} L(m), 0<a \leq 1,0 \leq m \leq M
$$




$$
L^{\prime}(m) \geq 0, \quad 0 \leq m \leq M .
$$

Condition (3.2) is called the Sölberg stability criterion ([35]).

\subsection{Compactness of Minimizing Sequence}

In this section, we first establish a compactness result for the minimizing sequences of the functional $F$. This compactness result is crucial for the existence and stability analyses.

Theorem 3.1. Suppose that the square of the angular momentum L satisfies (2.9), (3.1) and (3.2), and the pressure function p satisfies the following conditions

$$
p \in C^{1}[0,+\infty), \int_{0}^{1} \frac{p(\rho)}{\rho^{2}} d \rho<+\infty, \lim _{\rho \rightarrow \infty} \frac{p(\rho)}{\rho^{\gamma}}=K, p(\rho) \geq 0, p^{\prime}(\rho)>0 \text { for } \rho>0,
$$

where $0<K<+\infty$ and $\gamma \geq 4 / 3$. If

(1)

$$
\inf _{\rho \in W_{M}} F(\rho)<0
$$

and

(2) for $\rho \in W_{M}$,

$$
\int\left[A(\rho)(x)+\frac{1}{2} \frac{\rho(x) L\left(m_{\rho}(r(x))\right)}{r(x)^{2}}\right] d x \leq C_{1} F(\rho)+C_{2},
$$

for some positive constants $C_{1}$ and $C_{2}$, then the following hold:

(a) If $\left\{\rho^{i}\right\} \subset W_{M}$ is a minimizing sequence for the functional $F$, then there exist a sequence of vertical shifts $a_{i} \mathbf{e}_{\mathbf{3}}\left(a_{i} \in \mathbb{R}, \mathbf{e}_{\mathbf{3}}=(0,0,1)\right)$, a subsequence of $\left\{\rho^{i}\right\}$, (still labeled $\left.\left\{\rho^{i}\right\}\right)$, and a function $\tilde{\rho} \in W_{M}$, such that for any $\epsilon>0$ there exists $R>0$ with

$$
\int_{|x| \geq R} T \rho^{i}(x) d x \leq \epsilon, \quad i \in \mathbb{N}
$$

and

$$
T \rho^{i}(x) \rightarrow \tilde{\rho} \text {, weakly in } L^{\gamma}\left(\mathbb{R}^{3}\right) \text {, as } i \rightarrow \infty,
$$

where $T \rho^{i}(x):=\rho^{i}\left(x+a_{i} \mathbf{e}_{3}\right)$.

Moreover

(b)

$$
\nabla B\left(T \rho^{i}\right) \rightarrow \nabla B(\tilde{\rho}) \text { strongly in } L^{2}\left(\mathbb{R}^{3}\right) \text {, as } i \rightarrow \infty .
$$

(c) $\tilde{\rho}$ is a minimizer of $F$ in $W_{M}$.

Thus $\tilde{\rho}$ is a rotating star solution with total mass $M$ and angular momentum $\sqrt{L}$. 
Remark 2. i) The assumption (3.4) is crucial for our compactness and stability analysis. The physical meaning of this is that the gravitational energy, the negative part of the energy $F$, should be greater than the positive part, which means the gravitation should be strong enough to hold the star together. In section 4, we will verify this assumption. Roughly speaking, in addition to (3.3), if we require

$$
\lim _{\rho \rightarrow 0+} \frac{p(\rho)}{\rho^{\gamma_{1}}}=\alpha
$$

for some constants $\gamma_{1}>4 / 3$ and $0<\alpha<+\infty$, then (3.4) holds for the following cases:

(a) when $\gamma=4 / 3$ (where $\gamma$ is the constant in (3.3)), if the total mass $M$ is less than a "critical mass" $M_{c}$, then (3.4) holds. This case includes white dwarf stars. For a white dwarf star, $\gamma_{1}=5 / 3$.

(b) When $\gamma>4 / 3$, (3.4) holds for arbitrary positive total mass $M$. This generalizes our previous result in [28] for the polytropic stars with $p(\rho)=\rho^{\beta}, \beta>4 / 3$.

It should be noted that (3.9) does not apply to suppermassive star, i.e. $p(\rho)=k \rho^{4 / 3}$. For the supermassive star, in order that have that (3.4) hold, in additional to requiring that the total mass is less than a "critical mass", we also require that the angular momentum (per unit mass) $J$ is not identically zero.

ii) Assumption (2) in the above theorem implies that the functional $F$ is bounded below, i.e.,

$$
\inf _{\rho \in W_{M}} F(\rho)>-\infty
$$

We will verify this assumption in Section 4 (see Theorem 4.1).

iii) The inequality (3.6) is crucial for the compactness result (3.8). One of the difficulties in the analysis is the loss of compactness because we consider the problem in an unbounded space, $\mathbb{R}^{3}$. The inequality (3.6) means the masses of the elements in the minimizing sequence $T \rho^{i}(x)$ "almost" concentrate in a ball $B_{R}(0)$.

iv) It is easy to verify that the functional $F$ is invariant under any vertical shift, i.e., if $\rho(\cdot) \in W_{M}$, then $\bar{\rho}(x)=: \rho\left(x+a \mathbf{e}_{\mathbf{3}}\right) \in W_{M}$ and $F(\bar{\rho})=F(\rho)$ for any $a \in \mathbb{R}$. Therefore, if $\left\{\rho^{i}\right\}$ is a minimizing sequence of $F$ in $W_{M}$, then $\left\{T \rho^{i}\right\}=:=\rho^{i}\left(x+a_{i} \mathbf{e}_{\mathbf{3}}\right)$ is also a minimizing sequence in $W_{M}$.

Theorem 3.1 is proved in a sequence of lemmas with some modifications of the arguments in [28]. We only sketch the proofs of those lemmas and Theorem 3.1. Complete details can be followed as in [28]. We first give some inequalities which will be used later. We begin with Young's inequality (see [17], p. 146.)

Lemma 3.1. If $f \in L^{p} \cap L^{r}, 1 \leq p<q<r \leq+\infty$, then

$$
\|f\|_{q} \leq\|f\|_{p}^{a}\|f\|_{r}^{1-a}, \quad a=\frac{q^{-1}-r^{-1}}{p^{-1}-r^{-1}} .
$$


The following two lemmas are proved in [2].

Lemma 3.2. Suppose the function $f \in L^{1}\left(\mathbb{R}^{3}\right) \cap L^{q}\left(\mathbb{R}^{3}\right)$. If $1<q \leq 3 / 2$, then $B f=: f * \frac{1}{|x|}$ is in $L^{r}\left(\mathbb{R}^{3}\right)$ for $3<r<3 q /(3-2 q)$, and

$$
\|B f\|_{r} \leq C\left(\|f\|_{1}^{b}\|f\|_{q}^{1-b}+\|f\|_{1}^{c}\|f\|_{q}^{1-c}\right),
$$

for some constants $C>0,0<b<1$, and $0<c<1$. If $q>3 / 2$, then $B f(x)$ is a bounded continuous function, and satisfies (3.12) with $r=\infty$.

Lemma 3.3. For any function $f \in L^{1}\left(\mathbb{R}^{3}\right) \cap L^{4 / 3}\left(\mathbb{R}^{3}\right)$, if $\gamma \geq 4 / 3$, then $\nabla B f \in L^{2}\left(\mathbb{R}^{3}\right)$. Moreover,

$$
\left|\int f(x) B f(x) d x\right|=\frac{1}{4 \pi}\|\nabla B f\|_{2}^{2} \leq C\left(\int|f|^{4 / 3}(x) d x\right)\left(\int|f|(x) d x\right)^{2 / 3}
$$

for some constant $C$.

We also need the following lemma.

Lemma 3.4. Suppose that the pressure function p satisfies (3.3) and (3.5) holds. Let $\left\{\rho^{i}\right\} \subset$ $W_{M}$ be a minimizing sequence for the functional $F$. Then there exists a constant $C>0$ such that

$$
\int\left[\left(\rho^{i}\right)^{\gamma}(x)+\frac{1}{2} \frac{\rho^{i}(x) L\left(m_{\rho^{i}}(r(x))\right)}{r(x)^{2}}\right] d x \leq C, \text { for all } i \geq 1,
$$

where $\gamma \geq 4 / 3$ is the constant in (3.3). So, the sequence $\left\{\rho^{i}\right\}$ is bounded in $L^{\gamma}\left(\mathbb{R}^{3}\right)$.

Proof. By (3.5), we know that

$$
\int\left[A\left(\rho^{i}\right)(x)+\frac{1}{2} \frac{\rho^{i}(x) L\left(m_{\rho^{i}}(r(x))\right)}{r(x)^{2}}\right] d x \leq C, \text { for all } i \geq 1,
$$

for any minimizing sequence $\left\{\rho^{i}\right\} \subset W_{M}$ for the functional $F$, where we have used that $\left\{F\left(\rho^{i}\right)\right\}$ is bounded from above since it converges to $\inf _{W_{M}} F$. It is easy to verify that, by virtue of (3.3) and (2.5),

$$
\lim _{\rho \rightarrow \infty} \frac{A(\rho)}{\rho^{\gamma}}=\frac{K}{\gamma-1}, A(\rho)>0 \text { for } \rho>0 .
$$

Therefore, there exits a constant $\rho^{*}>0$ such that

$$
\alpha A(\rho) \geq \rho^{\gamma}, \quad \text { for } \rho \geq \rho^{*},
$$

where $\alpha=\frac{2(\gamma-1)}{K}$. Hence, for $\rho \in W_{M}$,

$$
\begin{aligned}
\int \rho^{\gamma} d x & \leq \int_{\rho<\rho^{*}}\left(\rho^{*}\right)^{\gamma-1} \rho d x+\alpha \int_{\rho \geq \rho^{*}} A(\rho) d x \\
& \leq\left(\rho^{*}\right)^{\gamma-1} M+\alpha \int A(\rho) d x .
\end{aligned}
$$

Applying this inequality to $\rho^{i}$, we conclude that the sequence $\left\{\rho^{i}\right\}$ is bounded in $L^{\gamma}\left(\mathbb{R}^{3}\right)$ by using (3.15). 
For any $M>0$, we let

$$
f_{M}=\inf _{\rho \in W_{M}} F(\rho) .
$$

Lemma 3.5. If (3.1) holds, then $f_{\bar{M}} \geq(\bar{M} / M)^{5 / 3} f_{M}$ for every $M>\bar{M}>0$.

Proof. The proof follows from a scaling argument as in [31] and [28]. Take $a=(M / \bar{M})^{1 / 3}$ and let $\bar{\rho}(x)=\rho(b x)$ for any $\rho \in W_{M}$. It is easy to verify that $\bar{\rho} \in W_{\bar{M}}$. Moreover, for $r \geq 0$, it is easy to verify, (as in [28]) that

$$
m_{\bar{\rho}}(r)=\frac{1}{a^{3}} m_{\rho}(a r) .
$$

Since $L$ satisfies (3.1) and $a>1$, we have

$$
L\left(m_{\bar{\rho}}(r)\right) \geq \frac{1}{a^{4}} L\left(m_{\rho}(b r)\right) .
$$

Thus, as in [28], we can show that

$$
\int \frac{\bar{\rho}(x) L\left(m_{\bar{\rho}}(r(x))\right)}{r(x)^{2}} d x=\frac{1}{a^{5}} \int \frac{\rho(x) L\left(m_{\bar{\rho}}(r(x))\right)}{r(x)^{2}} d x
$$

Therefore, since $a \geq 1$, it follows from (3.21) and (3.22) that

$$
\begin{aligned}
F(\bar{\rho}) & \geq a^{-3} \int A(\rho) d x-\frac{a^{-5}}{2} \int \rho B \rho d x+\frac{a^{-5}}{2} \int \frac{\rho(x) L\left(m_{\bar{\rho}}(r(x))\right)}{r(x)^{2}} d x \\
& \geq a^{-5}\left(\int A(\rho) d x-\frac{1}{2} \int \rho B \rho d x+\frac{1}{2} \int \frac{\rho(x) L\left(m_{\bar{\rho}}(r(x))\right)}{r(x)^{2}} d x\right) \\
& =(\bar{M} / M)^{5 / 3} F(\rho) .
\end{aligned}
$$

Since $\rho \rightarrow \bar{\rho}$ is one-to-one between $W_{M}$ and $W_{\bar{M}}$, this proves the lemma.

Lemma 3.6. Let $\left\{\rho^{i}\right\} \subset W_{M}$ be a minimizing sequence for $F$. Then there exist constants $r_{0}>0, \delta_{0}>0, i_{0} \in \mathbf{N}$ and $x^{i} \in \mathbb{R}^{3}$ with $r\left(x^{i}\right) \leq r_{0}$, such that

$$
\int_{B_{1}\left(x^{i}\right)} \rho^{i}(x) d x \geq \delta_{0}, \quad i \geq i_{0}
$$

Proof. First, since $\lim _{i \rightarrow \infty} F\left(\rho^{i}\right) \rightarrow f_{M}$ and $f_{M}<0$ (see (3.4)), for large $i$,

$$
-\frac{f_{M}}{2} \leq-F\left(\rho^{i}\right) \leq \frac{1}{2} \int \rho^{i} B \rho^{i} d x
$$

For any $i$, let

$$
\delta_{i}=\sup _{x \in \mathbb{R}^{3}} \int_{|y-x|<1} \rho^{i}(y) d y
$$


Now

$$
\begin{aligned}
& \int \rho^{i} B \rho^{i}(x) d x \\
& =\int_{\mathbb{R}^{3}} \rho^{i}(x)\left\{\int_{|y-x|<1}+\int_{1<|y-x|<r}+\int_{|y-x|>r}\right\} \frac{\rho^{i}(y)}{|y-x|} d y d x \\
& =: D_{1}+D_{2}+D_{3},
\end{aligned}
$$

and $D_{3} \leq M^{2} r^{-1}$. The shell $1<|y-x|<r$ can be covered by at most $C r^{3}$ balls of radius 1 , so $D_{2} \leq C M \delta_{i} r^{3}$. By using Hölder's inequality and applying (3.12) to the restriction of $\rho^{i}$ to $\{y:|y-x|<1\}$, we get

$$
\begin{aligned}
D_{1} & \leq\left\|\rho^{i}\right\|_{4 / 3}\left\|\int_{|y-x|<1} \frac{\rho^{i}(y)}{|y-x|} d y\right\|_{4} \\
& \leq C\left\|\rho^{i}\right\|_{4 / 3}\left(\left\|\chi_{B_{1}(x)} \rho^{i}\right\|_{1}^{b}\left\|\rho^{i}\right\|_{4 / 3}^{1-b}+\left\|\chi_{B_{1}(x)} \rho^{i}\right\|_{1}^{c}\left\|\rho^{i}\right\|_{4 / 3}^{1-c}\right) \\
& \leq C\left\|\rho^{i}\right\|_{4 / 3}\left(\delta_{i}^{b}\left\|\rho^{i}\right\|_{4 / 3}^{1-b}+\delta_{i}^{c}\left\|\rho^{i}\right\|_{4 / 3}^{1-c}\right)
\end{aligned}
$$

where $0<b<1$ and $0<c<1$. Now since $\left\{\left\|\rho^{i}\right\|_{\gamma}\right\}$ is bounded, it follows that $\left\{\left\|\rho^{i}\right\|_{4 / 3}\right\}$ is bounded due to the fact $\gamma \geq 4 / 3$ in view of (3.11) and $\left\|\rho^{i}\right\|_{1}=M$; this gives $D_{1} \leq C\left(\delta_{i}^{b}+\delta_{i}^{c}\right)$. It follows that we could choose $r$ so large that the above estimates give $\int \rho^{i} B \rho^{i}(x) d x<-f_{M}$ if $\delta_{i}$ were small enough. This would contradict (3.25). So there exists $\delta_{0}>0$ such that $\delta_{i} \geq \delta_{0}$ for large $i$. Thus, as $i$ is large, there exists $x^{i} \in \mathbb{R}^{3}$ and $i_{0} \in \mathbb{N}$ such that

$$
\int_{B_{1}\left(x^{i}\right)} \rho^{i}(x) d x \geq \delta_{0}, i \geq i_{0} .
$$

We now prove that there exists $r_{0}>0$ independent of $i$ such that $x^{i}$ must satisfy $r\left(x^{i}\right) \leq r_{0}$ for $i$ large. Namely, since $\rho^{i}$ has mass at least $\delta_{0}$ in the unit ball centered at $x^{i}$, and is axially symmetric, it has mass $\geq \operatorname{Cr}\left(x^{i}\right) \delta_{0}$ in the torus obtained by revolving this ball around $x_{3}$-axis (or $z$-axis).Therefore $r\left(x^{i}\right) \leq\left(C \delta_{0}\right)^{-1} M$.

In order to prove Theorem 3.1, we will need the following lemma.

Lemma 3.7. Let $\left\{f^{i}\right\}$ be a bounded sequence in $L^{\gamma}\left(\mathbb{R}^{3}\right)(\gamma \geq 4 / 3)$ and suppose

$$
f^{i} \rightarrow f^{0} \text { weakly in } L^{\gamma}\left(\mathbb{R}^{3}\right)
$$

Then

(a) For any $R>0$,

$$
\nabla B\left(\chi_{B_{R}(0)} f^{i}\right) \rightarrow \nabla B\left(\chi_{B_{R}(0)} f^{0}\right) \text { strongly in } L^{2}\left(\mathbb{R}^{3}\right)
$$

where $\chi$ is the indicator function.

(b) If in addition $\left\{f^{i}\right\}$ is bounded in $L^{1}\left(\mathbb{R}^{3}\right), f^{0} \in L^{1}\left(\mathbb{R}^{3}\right)$, and for any $\epsilon>0$ there exist 
$R>0$ and $i_{0} \in \mathbf{N}$ such that

$$
\int_{|x|>R}\left|f^{i}(x)\right| d x<\epsilon, \quad i \geq i_{0}
$$

then

$$
\nabla B f^{i} \rightarrow \nabla B f^{0} \text { strongly in } L^{2}\left(\mathbb{R}^{3}\right) .
$$

Proof. This lemma follows easily from the proof of Lemma 3.7 in [31], due to the following observation:

The map: $\rho \in L^{\gamma}\left(\mathbb{R}^{3}\right) \mapsto I_{B_{R}(0)} \nabla B\left(I_{B_{R}(0)} \rho\right)$ is compact for any $R>0$, if $\gamma \geq 4 / 3$.

With above lemmas, the proof of Theorem 3.1] is similar to that in [28]. So we only outline the main steps.

Proof of Theorem 3.1

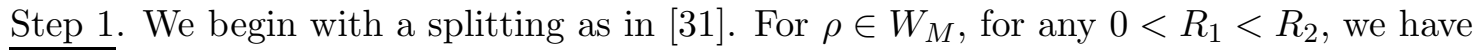

$$
\rho=\rho \chi_{|x| \leq R_{1}}+\rho \chi_{R_{1}<|x| \leq R_{2}}+\rho \chi_{|x|>R_{2}}=: \rho_{1}+\rho_{2}+\rho_{3},
$$

where $\chi$ is the indicator function. It is easy to verify that

$$
\begin{aligned}
\int \frac{\rho(x) L\left(m_{\rho}(r(x))\right.}{r^{2}(x)} d x & =\sum_{j=1}^{3} \int \frac{\rho_{j}(x) L\left(m_{\rho_{j}}(r(x))\right.}{r^{2}(x)} d x \\
& +\sum_{j=1}^{3} \int \frac{\rho_{j}(x)\left(L \left(m_{\rho}(r(x))-L\left(m_{\rho_{j}}(r(x))\right.\right.\right.}{r^{2}(x)} d x \\
& \geq \sum_{j=1}^{3} \int \frac{\rho_{j}(x) L\left(m_{\rho_{j}}(r(x))\right.}{r^{2}(x)} d x .
\end{aligned}
$$

In the last inequality above, we have used (3.2). So, we have

$$
F(\rho) \geq \sum_{j=1}^{3} F\left(\rho_{j}\right)-\sum_{1 \leq i<j \leq 3} I_{i j}
$$

where

$$
I_{i j}=\int_{\mathbb{R}^{3}} \int_{\mathbb{R}^{3}}|x-y|^{-1} \rho_{i}(x) \rho_{j}(y) d x d y, \quad 1 \leq i<j \leq 3 .
$$

If we choose $R_{2}>2 R_{1}$ in the splitting (3.32), then

$$
I_{13} \leq \frac{C}{R_{2}}
$$

By (3.12) and (3.13), we have

$$
\begin{aligned}
& I_{12}+I_{23} \\
& =\frac{1}{4 \pi} \int \nabla\left(B \rho_{1}+B \rho_{3}\right) \cdot \nabla B \rho_{2} d x \leq C\left\|\nabla\left(B \rho_{1}+B \rho_{3}\right)\right\|_{2}\left\|\nabla B \rho_{2}\right\|_{2} \\
& \leq C M^{1 / 3}\left\|\rho_{1}+\rho_{3}\right\|_{4 / 3}^{2 / 3}\left\|\nabla B \rho_{2}\right\| 2 \leq C M^{1 / 3}\|\rho\|_{4 / 3}^{2 / 3}\left\|\nabla B \rho_{2}\right\|_{2} .
\end{aligned}
$$


Using Lemma 3.5, (3.4), (3.34), (3.35) and (3.36), and following an argument as in the proof of Theorem 3.1 in [31], we can show that

$$
\begin{aligned}
& f_{M}-F(\rho) \\
& \leq\left(1-\left(\frac{M_{1}}{M}\right)^{5 / 3}-\left(\frac{M_{2}}{M}\right)^{5 / 3}-\left(\frac{M_{3}}{M}\right)^{5 / 3}\right) f_{M}+C\left(R_{2}^{-1}+M^{1 / 3}\|\rho\|_{4 / 3}^{2 / 3}\left\|\nabla B \rho_{2}\right\|_{2}\right) \\
& \leq C f_{M} M_{1} M_{3}+C\left(R_{2}^{-1}+M^{1 / 3}\|\rho\|_{4 / 3}^{2 / 3}\left\|\nabla B \rho_{2}\right\|_{2}\right),
\end{aligned}
$$

by choosing $R_{2}>2 R_{1}$ in the splitting (3.32), where $M_{i}=\int \rho_{i}(x) d x\left(i=1,2,3\right.$.) Let $\left\{\rho^{i}\right\}$ be a minimizing sequence of $F$ in $W_{M}$. By Lemma 3.6, we know that there exists $i_{0} \in \mathbf{N}$ and $\delta_{0}>0$ independent of $i$ such that

$$
\int_{a_{i} \mathbf{e}_{3}+B_{R_{0}(0)}} \rho^{i}(x) d x \geq \delta_{0}, \quad \text { if } i \geq i_{0},
$$

where $a_{i}=z\left(x^{i}\right)$ and $R_{0}=r_{0}+1, x^{i}$ and $r_{0}$ are those quantities in Lemma 3.6 , $\mathbf{e}_{\mathbf{3}}=(0,0,1)$. Having proved (3.38), we can follow the argument in the proof of Theorem 3.1 in [31] to verify (3.31) for

$$
f^{i}(x)=T \rho^{i}(x)=: \rho^{i}\left(\cdot+a_{i} \mathbf{e}_{3}\right)
$$

by using (3.34) and (3.38) and choosing suitable $R_{1}$ and $R_{2}$ in the splitting (3.32). We sketch this as follows. The sequence $T \rho^{i}=: \rho^{i}\left(\cdot+a_{i} \mathbf{e}_{\mathbf{3}}\right), i \geq i_{0}$, is a minimizing sequence of $F$ in $W_{M}$ (see Remark 2 after Theorem 3.1). We rewrite (3.38) as

$$
\int_{B_{R_{0}}(0)} T \rho^{i}(x) d x \geq \delta_{0}, i \geq i_{0}
$$

Applying (3.37) with $T \rho^{i}$ replacing $\rho$, and noticing that $\left\{T \rho^{i}\right\}$ is bounded in $L^{\gamma}\left(\mathbb{R}^{3}\right.$ ) (see Lemma 3.4) (so $\left\{\left\|T \rho^{i}\right\|_{4 / 3}\right\}$ is bounded if $\gamma \geq 4 / 3$ in view of (3.11) and the fact $\left\|\rho^{i}\right\|_{1}=M$ ), we obtain, if $R_{2}>2 R_{1}$,

$$
-C f_{M} M_{1}^{i} M_{3}^{i} \leq C\left(R_{2}^{-1}+\left\|\nabla B T \rho_{2}^{i}\right\|_{2}\right)+F\left(T \rho^{i}\right)-f_{M},
$$

where $M_{1}^{i}=\int T \rho_{1}^{i}(x) d x=\int_{|x|<R_{1}} T \rho^{i}(x) d x, M_{3}^{i}=\int T \rho_{3}^{i}(x) d x=\int_{|x|>R_{2}} T \rho^{i}(x) d x$ and $T \rho_{2}^{i}=\chi_{R_{1}<|x| \leq R_{2}} T \rho^{i}$. Since $\left\{T \rho^{i}\right\}$ is bounded in $L^{\gamma}\left(\mathbb{R}^{3}\right)$, there exists a subsequence, still labeled by $\left\{T \rho^{i}\right\}$, and a function $\tilde{\rho} \in W_{M}$ such that

$$
T \rho^{i} \rightarrow \tilde{\rho} \text { weakly in } L^{\gamma}\left(\mathbb{R}^{3}\right)
$$

This proves (3.7). By (3.39), we know that $M_{1}^{i}$ in (3.40) satisfies $M_{1}^{i} \geq \delta_{0}$ for $i \geq i_{0}$ by choosing $R_{1} \geq R_{0}$ where $R_{0}$ is the constant in (3.39). Therefore, by (3.40) and the fact that $f_{M}<0$ (cf. (3.4) ) , we have

$$
\left.-C f_{M} \delta_{0} M_{3}^{i} \leq C R_{2}^{-1}+C\left\|\nabla B \tilde{\rho}_{2}\right\|_{2}+C\left\|\nabla B T \rho_{2}^{i}-\nabla B \tilde{\rho}_{2}\right\|_{2}\right)+F\left(T \rho^{i}\right)-f_{M},
$$


where $\tilde{\rho}_{2}=\chi_{|x|>R_{2}} \tilde{\rho}$. Given any $\epsilon>0$, by the same argument as [31, we can increase $R_{1}>R_{0}$ such that the second term on the right hand side of (3.41) is small, say less than $\epsilon / 4$. Next choose $R_{2}>2 R_{1}$ such that the first term is small. Now that $R_{1}$ and $R_{2}$ are fixed, the third term on the right hand side of (3.41) converges to zero by Lemma 3.7(a). Since $\left\{T \rho^{i}\right\}$ is a minimizing sequence of $F$ in $W_{M}$, we can make $F\left(T \rho^{i}\right)-f_{M}$ small by taking $i$ large. Therefore, for $i$ sufficiently large, we can make

$$
M_{3}^{i}=: \int_{|x|>R_{2}} T \rho^{i}(x) d x<\epsilon
$$

This verifies (3.31) in Lemma 3.7 for $f^{i}=T \rho^{i}$. By weak convergence we have that for any $\epsilon>0$ there exists $R>0$ such that

$$
M-\epsilon \leq \int_{B_{R}(0)} \tilde{\rho}(x) d x \leq M,
$$

which implies $\tilde{\rho} \in L^{1}\left(\mathbb{R}^{3}\right)$ with $\int \tilde{\rho} d x=M$. Therefore, by Lemma 3.7(b), we have

$$
\left\|\nabla B T \rho^{i}-\nabla B \tilde{\rho}\right\|_{2} \rightarrow 0, \quad i \rightarrow+\infty
$$

This proves (3.8). (3.6) in Theorem 3.1 follows from (3.42) by taking $R=R_{2}$.

Let $\left\{\rho^{i}\right\}$ be a minimizing sequence of the energy functional $F$, and let $\tilde{\rho}$ be a weak limit of $\left\{T \rho^{i}\right\}$ in $L^{\gamma}\left(\mathbb{R}^{3}\right)$. We will prove that $\tilde{\rho}$ is a minimizer of $F$ in $W_{M}$; that is

$$
F(\tilde{\rho}) \leq \lim \inf _{i \rightarrow \infty} F\left(T \rho^{i}\right)
$$

By (3.3), there exist positive constants $C$ and $\rho^{*}$ such that

$$
A^{\prime}(\rho) \leq C \rho^{\gamma-1}, \text { for } \rho \geq \rho^{*}
$$

where $\gamma \geq 4 / 3$ is the constant in (3.3). Since $\tilde{\rho} \in L^{\gamma}$ and $\int \tilde{\rho} d x=M$, we can conclude $A^{\prime}(\tilde{\rho}) \in L^{\gamma^{\prime}}$, where $L^{\gamma^{\prime}}$ is the dual space of $L^{\gamma}$, i.e., $\gamma^{\prime}=\frac{\gamma}{\gamma-1}$. In view of (2.5) and (3.3), we have

$$
A^{\prime \prime}(\rho)=p^{\prime}(\rho) / \rho>0, \quad \text { for } \rho>0,
$$

so that

$$
\int A\left(T \rho^{i}\right) d x \geq \int A(\tilde{\rho}) d x+\int A^{\prime}(\tilde{\rho})\left(T \rho^{i}-\tilde{\rho}\right), \text { for } i \geq 1 .
$$

Since $A^{\prime}(\tilde{\rho}) \in L^{\gamma^{\prime}}$ and $T \rho^{i}$ weakly converges to $\tilde{\rho}$ in $L^{\gamma}$,

$$
\int A^{\prime}(\tilde{\rho})\left(T \rho^{i}-\tilde{\rho}\right) \rightarrow 0, \text { as } i \rightarrow+\infty
$$

Therefore,

$$
\int A(\tilde{\rho}) d x \leq \lim \inf _{i \rightarrow \infty} \int A\left(T \rho^{i}\right) d x .
$$


Next, following the proof in 28 , we can show that

$$
\lim _{i \rightarrow \infty} \inf \int \frac{T \rho^{i}(x) L\left(m_{T \rho^{i}}(r(x))-\tilde{\rho}(x) L\left(m_{\tilde{\rho}}(r(x))\right.\right.}{r^{2}(x)} d x \geq 0,
$$

by showing that the mass function

$$
m_{\tilde{\rho}}(r)=: \int_{\sqrt{x_{1}^{2}+x_{2}^{2}} \leq r} \tilde{\rho}(x) d x
$$

is continuous for $r \geq 0$, and using (3.6). Then (3.44) follows from (3.43), (3.49) and (3.50).

\section{$3.2 \quad$ Stability}

In this section, we assume that the pressure function $p$ satisfies

$$
p \in C^{1}[0,+\infty), \lim _{\rho \rightarrow 0+} \frac{p(\rho)}{\rho^{6 / 5}}=0, \lim _{\rho \rightarrow \infty} \frac{p(\rho)}{\rho^{\gamma}}=K, p^{\prime}(\rho)>0 \text { for } \rho>0 .
$$

where $0<K<+\infty$ and $\gamma \geq 4 / 3$ are constants. It should be noticed that (3.51) implies both (2.15) and (3.3). We consider the Cauchy problem for (1.1) with the initial data

$$
\rho(x, 0)=\rho_{0}(x), \mathbf{v}(x, 0)=\mathbf{v}_{0}(x) .
$$

We begin by giving the definition of a weak solution.

Definition: Let $\rho \mathbf{v}=\mathbf{m}$. The triple $(\rho, \mathbf{m}, \Phi)(x, t)\left(x \in \mathbb{R}^{3}, t \in[0, T]\right)(T>0)$ and $\Phi$ given by (1.2), with $\rho \geq 0, \mathbf{m}, \mathbf{m} \otimes \mathbf{m} / \rho$ and $\rho \nabla \Phi$ being in $L_{\text {loc }}^{1}\left(\mathbb{R}^{3} \times[0, T]\right)$, is called a weak solution of the Cauchy problem (1.1) and (3.52) on $\mathbb{R}^{3} \times[0, T]$ if for any Lipschitz continuous test functions $\psi$ and $\mathbf{\Psi}=\left(\psi_{1}, \psi_{2}, \psi_{3}\right)$ with compact supports in $\mathbb{R}^{3} \times[0, T]$,

$$
\int_{0}^{T} \int\left(\rho \psi_{t}+\mathbf{m} \cdot \nabla \psi\right) d x d t+\int \rho_{0}(x) \psi(x, 0) d x=0
$$

and

$$
\int_{0}^{T} \int\left(\mathbf{m} \cdot \mathbf{\Psi}_{t}+\frac{\mathbf{m} \otimes \mathbf{m}}{\rho} \cdot \nabla \mathbf{\Psi}\right) d x d t+\int \mathbf{m}_{0}(x) \mathbf{\Psi}(x, 0) d x=\int_{0}^{T} \int \rho \nabla \Phi \mathbf{\Psi} d x d t
$$

both hold.

For any weak solution, it is easy to verify that the total mass is conserved by using a generalized divergence theorem for $L^{r}$ functions $(r \geq 1)$ (cf. [8]),

$$
\int \rho(x, t) d x=\int \rho(x, 0) d x, \quad t \geq 0 .
$$

The total energy of system (1.1) at time $t$ is

$$
E(t)=E(\rho(t), \mathbf{v}(t))=\int\left(A(\rho)+\frac{1}{2} \rho|\mathbf{v}|^{2}\right)(x, t) d x-\frac{1}{8 \pi} \int|\nabla \Phi|^{2}(x, t) d x
$$


where as before,

$$
A(\rho)=\rho \int_{0}^{\rho} \frac{p(s)}{s^{2}} d s .
$$

For a solution of (1.1) without shock waves, the total energy is conserved, i.e., $E(t)=E(0)$ $(t \geq 0)$ (cf. [35]). For solutions with shock waves, the energy should be non-increasing in time, so that for all $t \geq 0$,

$$
E(t) \leq E(0)
$$

due to the entropy conditions, which are motivated by the second law of thermodynamics (cf. [20] and [34]). This was proved in [28].

We consider axi-symmetric initial data, which takes the form

$$
\begin{aligned}
& \rho_{0}(x)=\rho(r, z), \\
& \mathbf{v}_{0}(x)=v_{0}^{r}(r, z) \mathbf{e}_{r}+v_{0}^{\theta}(r, z) \mathbf{e}_{\theta}+v_{0}^{3}(\rho, z) \mathbf{e}_{3} .
\end{aligned}
$$

Here $r=\sqrt{x_{1}^{2}+x_{2}^{2}}, z=x_{3}, x=\left(x_{1}, x_{2}, x_{3}\right) \in \mathbb{R}^{3}$ (as before), and

$$
\mathbf{e}_{r}=\left(x_{1} / r, x_{2} / r, 0\right)^{\mathrm{T}}, \mathbf{e}_{\theta}=\left(-x_{2} / r, x_{1} / r, 0\right)^{\mathrm{T}}, \mathbf{e}_{3}=(0,0,1)^{\mathrm{T}} .
$$

We seek axi-symmetric solutions of the form

$$
\begin{aligned}
& \rho(x, t)=\rho(r, z, t), \\
& \mathbf{v}(x, t)=v^{r}(r, z, t) \mathbf{e}_{r}+v^{\theta}(r, z, t) \mathbf{e}_{\theta}+v^{3}(r, z, t) \mathbf{e}_{3}, \\
& \Phi(x, t)=\Phi(r, z, t)=-B \rho(r, z, t),
\end{aligned}
$$

We call a vector field $\mathbf{u}(x, t)=\left(u_{1}, u_{2}, u_{3}\right)(x)\left(x \in \mathbb{R}^{3}\right)$ axi-symmetric if it can be written in the form

$$
\mathbf{u}(x)=u^{r}(r, z) \mathbf{e}_{r}+u^{\theta}(r, z) \mathbf{e}_{\theta}+u^{3}(\rho, z) \mathbf{e}_{3} .
$$

For the velocity field $\mathbf{v}=\left(v_{1}, v_{2}, v_{3}\right)(x, t)$, we define the angular momentum (per unit mass) $j(x, t)$ about the $x_{3}$-axis at $(x, t), t \geq 0$, by

$$
j(x, t)=x_{1} v_{2}-x_{2} v_{1} .
$$

For an axi-symmetric velocity field

$$
\begin{gathered}
\mathbf{v}(x, t)=v^{r}(r, z, t) \mathbf{e}_{r}+v^{\theta}(r, z, t) \mathbf{e}_{\theta}+v^{3}(\rho, z, t) \mathbf{e}_{3} \\
v_{1}=\frac{x_{1}}{r} v^{r}-\frac{x_{2}}{r} v^{\theta}, v_{2}=\frac{x_{2}}{r} v^{r}+\frac{x_{1}}{r} v^{\theta}, v_{3}=v^{3}
\end{gathered}
$$

so that

$$
j(x, t)=r v^{\theta}(r, z, t) .
$$

In view of (3.64) and (3.66), we have

$$
|\mathbf{v}|^{2}=\left|v^{r}\right|^{2}+\frac{j^{2}}{r^{2}}+\left|v^{3}\right|^{2} .
$$


Therefore, the total energy at time $t$ can be written as

$$
\begin{aligned}
E(\rho(t), \mathbf{v}(t)) & =\int A(\rho)(x, t) d x+\frac{1}{2} \int \frac{\rho j^{2}(x, t)}{r^{2}(x)} d x \\
& -\frac{1}{8 \pi} \int|\nabla B \rho|^{2}(x, t) d x+\frac{1}{2} \int \rho\left(\left|v^{r}\right|^{2}+\left|v^{3}\right|^{2}\right)(x, t) d x .
\end{aligned}
$$

There are two important conserved quantities for the Euler-Poisson equations (1.1); namely the total mass and the angular momentum. In order to describe these, we define $D_{t}$, the nonvacuum region at time $t \geq 0$ of the solution by

$$
D_{t}=\left\{x \in \mathbb{R}^{3}: \rho(x, t)>0\right\} .
$$

We will make the following physically reasonable assumptions A1)-A4) on weak solutions of the Cauchy problem (1.1) and (3.52) (A1)-A4) are easily verified for smooth solutions. For general weak solutions, they are motivated by physical considerations, cf. [35]).

A1) For any $t \geq 0$, there exists a measurable subset $G_{t} \subset D_{t}$ with meas $\left(D_{t}-G_{t}\right)=0$ (meas denotes Lebsegue measure) such that, for any $x \in G_{t}$, there exists a unique (backwards) particle path $\xi(\tau, x, t)$ for $0 \leq \tau \leq t$ satisfying

$$
\partial_{\tau} \xi(\tau, x, t)=\mathbf{v}(\xi(\tau, x, t), \tau), \xi(t, x, t)=x .
$$

Remark 3. If $\mathbf{v}(\cdot, t) \in B V\left(\mathbb{R}^{3}\right)$ and $\operatorname{div}_{x} v(\cdot, t) \in L^{\infty}\left(\mathbb{R}^{3}\right)$ for $t \geq 0\left(\operatorname{div}_{x}\right.$ is in the sense of distributions), it was proved by L. Ambrosio ([1]) that A1) is valid. Related results can be found in [9].

For $x \in G_{t}$, we write

$$
\xi(0, x, t)=\xi_{-t}(x)
$$

Also, for $x \in \mathbb{R}^{3}$ and $t \geq 0$, we denote the total mass at time $t$ in the cylinder $\left\{y \in \mathbb{R}^{3}\right.$ : $r(y) \leq r(x)\}$ by $m_{\rho(t)}(r(x))$, i.e.,

$$
m_{\rho(t)}(r(x))=\int_{r(y) \leq r(x)} \rho(y, t) d y .
$$

For axi-symmetric motion, we assume

A2)

$$
m_{\rho(t)}(r(x))=m_{\rho_{0}}\left(r\left(\xi_{-t}(x)\right)\right), \quad \text { for } x \in G_{t}, t \geq 0 .
$$

(This means that the mass enclosed within any material volume cannot change as we follow the volume in its motion ( [35], p. 47)). Moreover, we assume that the angular momentum is conserved along the particle path:

A3)

$$
j(x, t)=j\left(\xi_{-t}(x), 0\right), \quad \text { for } x \in G_{t}, t \geq 0 .
$$


Finally, for $L=j^{2}$, we need a technical assumption; namely,

A4)

$$
\lim _{r \rightarrow 0+} \frac{L\left(m_{\rho(t)}(r)+m_{\tilde{\rho}}(r)\right) m_{\sigma(t)}(r)}{r^{2}}=0
$$

for $t \geq 0$, where $\sigma(t)=\rho(t)-\tilde{\rho}$.

Remark 4. (3.74) can be understood as follows. For any $\rho \in W_{M}$, we have $\lim _{r \rightarrow 0+} m_{\rho}(r)=0$. Therefore $\lim _{r \rightarrow 0+} L\left(m_{\rho(t)}(r)+m_{\tilde{\rho}}(r)\right)=L(0)=0$, so if we define

$$
\hat{\rho}(s, t)-\hat{\tilde{\rho}} \rho(s)=\int_{-\infty}^{+\infty}(\rho(s, z, t)-\tilde{\rho}(s, z)) d z
$$

then if

$$
\frac{m_{\sigma(t)}(r)}{r^{2}}=\frac{\int_{0}^{r}(2 \pi s(\hat{\rho}(s, t)-\hat{\tilde{\rho}}(s)) d s}{r^{2}} \in L^{\infty}(0, \delta) \text { for some } \delta>0,
$$

(3.74) will hold. If $\hat{\rho}(\cdot, t)-\hat{\tilde{\rho}}(\cdot) \in L^{\infty}(0, \delta)$, then (3.75) holds. This can be assured by assuming that $\rho(r, z, t)-\tilde{\rho}(r, z) \in L^{\infty}\left((0, \delta) \times \mathbb{R} \times \mathbb{R}^{+}\right)$and decays fast enough in the $z$ direction. For example, when $\rho(x, t)-\tilde{\rho}(x)$ has compact support in $\mathbb{R}^{3}$ and $\rho(\cdot, t)-\tilde{\rho}(\cdot) \in L^{\infty}\left(\mathbb{R}^{3}\right)$, then (3.74) holds.

We next make some assumptions on the initial data; namely, we assume that the initial data is such that the initial total mass and angular momentum are the same as those of the rotating star solution (those two quantities are conserved quantities). Therefore, we require

$\left.\mathrm{I}_{1}\right)$

$$
\int \rho_{0}(x) d x=\int \tilde{\rho}(x) d x=M
$$

Moreover we assume

$\left.\mathrm{I}_{2}\right)$ For the initial angular momentum $j(x, 0)=r v_{0}^{\theta}(r, z)=: j_{0}(r, z)\left(r=\sqrt{x_{1}^{2}+x_{2}^{2}}, z=x_{3}\right.$ for $x=\left(x_{1}, x_{2}, x_{3}\right)$, we assume $j(x, 0)$ only depends on the total mass in the cylinder $\{y \in$ $\left.\mathbb{R}^{3}, r(y) \leq r(x)\right\}$, i.e. ,

$$
j(x, 0)=j_{0}\left(m_{\rho_{0}}(r(x))\right) .
$$

(This implies that we require that $v_{0}^{\theta}(r, z)$ only depends on $r$.)

Finally, we assume that the initial profile of the angular momentum per unit mass is the same as that of the rotating star solution, i. e.,

$\left.\mathrm{I}_{3}\right)$

$$
j_{0}^{2}(m)=L(m), \quad 0 \leq m \leq M,
$$

where $L(m)$ is the profile of the square of the angular momentum of the rotating star defined in Section 2. 
In order to state our stability result, we need some notation. Let $\lambda$ be the constant in Theorem 2.2 , i.e.,

$$
\left\{\begin{array}{l}
A^{\prime}(\tilde{\rho}(x))+\int_{r(x)}^{\infty} L\left(m_{\tilde{\rho}}(s)\right) s^{-3} d s-B \tilde{\rho}(x)=\lambda, x \in \Gamma, \\
\left.\int_{r(x)}^{\infty} L\left(m_{\tilde{\rho}}\right)(s)\right) s^{-3} d s-B \tilde{\rho}(x) \geq \lambda, \quad x \in \mathbb{R}^{3}-\Gamma,
\end{array}\right.
$$

with $A$ defined in (3.57) and $\Gamma$ defined in (2.16).

For $\rho \in W_{M}$, we define,

$$
d(\rho, \tilde{\rho})=\int[A(\rho)-A(\tilde{\rho})]+(\rho-\tilde{\rho}) \int_{r(x)}^{\infty}\left\{\frac{L\left(m_{\tilde{\rho}}(s)\right)}{s^{3}} d s-\lambda-B \tilde{\rho}\right\} d x .
$$

For $x \in \Gamma$, in view of the convexity of the function $A$ (cf. (3.46)) and (3.79), we have,

$$
\begin{aligned}
& (A(\rho)-A(\tilde{\rho}))(x)+\left(\int_{r(x)}^{\infty} \frac{L\left(m_{\tilde{\rho}}(s)\right)}{s^{3}} d s-\lambda-B \tilde{\rho}(x)\right)(\rho-\tilde{\rho}) \\
& =\left(A(\rho)-A(\tilde{\rho})-A^{\prime}(\tilde{\rho})(\rho-\tilde{\rho})\right)(x) \geq 0 .
\end{aligned}
$$

For $x \in \mathbb{R}^{3}-\Gamma, \tilde{\rho}(x)=0$, so we have $\left.A(\tilde{\rho})(x)\right)=0$. This is because since $A(0)=0$ due to $p(0)=0$ (cf. (3.3)) and (2.5). Therefore, by (3.79), we have, for $\rho \in W_{M}$ and $x \in \mathbb{R}^{3}-\Gamma$,

$$
\begin{aligned}
& (A(\rho)-A(\tilde{\rho}))(x)+\left(\int_{r(x)}^{\infty} \frac{L\left(m_{\tilde{\rho}}(s)\right)}{s^{3}} d s-\lambda-B \tilde{\rho}(x)\right)(\rho-\tilde{\rho}) \\
& =A(\rho) \geq 0 .
\end{aligned}
$$

Thus, for $\rho \in W_{M}$,

$$
d(\rho, \tilde{\rho}) \geq 0
$$

We also define

$$
\begin{aligned}
d_{1}(\rho, \tilde{\rho}) & =\frac{1}{2} \int \frac{\rho(x) L\left(m_{\rho}(r(x))-\tilde{\rho}(x) L\left(m_{\tilde{\rho}}(r(x))\right.\right.}{r^{2}(x)} d x \\
& -\iint_{r(x)}^{\infty} s^{-3} L\left(m_{\tilde{\rho}}(s)\right) d s(\rho(x)-\tilde{\rho}(x)) d x
\end{aligned}
$$

for $\rho \in W_{M}$. We shall show later that $d_{1} \geq 0$. Our main stability result in this paper is the following global-in-time stability theorem.

Theorem 3.2. Suppose that the pressure function satisfies (3.51), and both (3.4), (3.5) hold. Let $\tilde{\rho}$ be a minimizer of the functional $F$ in $W_{M}$, and assume that it is unique up to a vertical shift. Assume that $\left.\left.I_{1}\right)-I_{3}\right)$, [(3.76)- (3.78)] hold. Moreover, assume that the angular momentum of the rotating star solution $\tilde{\rho}$ satisfies (2.9), (3.1) and (3.2). Let $(\rho, \mathbf{v}, \Phi)(x, t)$ be an axi-symmetric weak solution of the Cauchy problem (1.1) and (3.52) satisfying the 
assumptions A1)-A4), [(3.70)-(3.74)]. If the total energy $E(t)$ (cf. (3.56)) is non-increasing with respect to $t$, then for every $\epsilon>0$, there exists a number $\delta>0$ such that if

$$
\begin{aligned}
& d\left(\rho_{0}, \tilde{\rho}\right)+\frac{1}{8 \pi} \| \nabla B \rho_{0}-\nabla B \tilde{\rho}||_{2}^{2}+\left|d_{1}\left(\rho_{0}, \tilde{\rho}\right)\right| \\
& +\frac{1}{2} \int \rho_{0}(x)\left(\left|v_{0}^{r}\right|^{2}+\left|v_{0}^{3}\right|^{2}\right)(x) d x<\delta,
\end{aligned}
$$

then there is a vertical shift $a \mathbf{e}_{\mathbf{3}}\left(a \in \mathbb{R}, \mathbf{e}_{\mathbf{3}}=(0,0,1)\right)$ such that, for every $t>0$

$$
\begin{aligned}
& d\left(\rho(t), T^{a} \tilde{\rho}\right)+\frac{1}{8 \pi} \| \nabla B \rho(t)-\nabla B T^{a} \tilde{\rho}||_{2}^{2}+\left|d_{1}\left(\rho(t), T^{a} \tilde{\rho}\right)\right| \\
& +\frac{1}{2} \int \rho(x, t)\left(\left|v^{r}(x, t)\right|^{2}+\left|v^{3}(x, t)\right|^{2}\right) d x<\epsilon,
\end{aligned}
$$

where $T^{a} \tilde{\rho}(x)=: \tilde{\rho}\left(x+a \mathbf{e}_{3}\right)$.

Remark 5. As noted in [28], the vertical shift $a \mathbf{e}_{\mathbf{3}}$ appearing in the theorem is analogous to a similar phenomenon which appears in the study of stability of viscous traveling waves in conservation laws, whereby convergence is to a "shift" of the original traveling wave.

Remark 6. Without the uniqueness assumption for the minimizer of $F$ in $W_{M}$, we can have the following type of stability result, as observed in [32] for the non-rotating star solutions. Suppose the assumptions in Theorem 3.2 hold. Let $\mathcal{S}_{M}$ be the set of all minimizers of $F$ in $W_{M}$ and $(\rho, \mathbf{v}, \Phi)(x, t)$ be an axi-symmetric weak solution of the Cauchy problem (1.1) and (3.52). If the total energy $E(t)$ is non-increasing with respect to $t$, then for every $\epsilon>0$, there exists a number $\delta>0$ such that if

$$
\begin{aligned}
& \inf _{\tilde{\rho} \in \mathcal{S}_{M}}\left[d\left(\rho_{0}, \tilde{\rho}\right)+\frac{1}{8 \pi}|| \nabla B \rho_{0}-\nabla B \tilde{\rho}||_{2}^{2}+\left|d_{1}\left(\rho_{0}, \tilde{\rho}\right)\right|\right] \\
& +\frac{1}{2} \int \rho_{0}(x)\left(\left|v_{0}^{r}\right|^{2}+\left|v_{0}^{3}\right|^{2}\right)(x) d x<\delta
\end{aligned}
$$

then for every $t>0$

$$
\begin{aligned}
& \inf _{\tilde{\rho} \in \mathcal{S}_{M}}\left[d\left(\rho(t), T^{a} \tilde{\rho}\right)+\frac{1}{8 \pi}|| \nabla B \rho(t)-\nabla B T^{a} \tilde{\rho} \|_{2}^{2}+\left|d_{1}\left(\rho(t), T^{a} \tilde{\rho}\right)\right|\right] \\
& +\frac{1}{2} \int \rho(x, t)\left(\left|v^{r}(x, t)\right|^{2}+\left|v^{3}(x, t)\right|^{2}\right)(x) d x<\epsilon .
\end{aligned}
$$

then there is a vertical shift $a \mathbf{e}_{\mathbf{3}}\left(a \in \mathbb{R}, \mathbf{e}_{\mathbf{3}}=(0,0,1)\right)$ such that, for every $t>0$

$$
\begin{aligned}
& d\left(\rho(t), T^{a} \tilde{\rho}\right)+\frac{1}{8 \pi} \| \nabla B \rho(t)-\nabla B T^{a} \tilde{\rho}||_{2}^{2}+\left|d_{1}\left(\rho(t), T^{a} \tilde{\rho}\right)\right| \\
& +\frac{1}{2} \int \rho(x, t)\left(\left|v^{r}(x, t)\right|^{2}+\left|v^{3}(x, t)\right|^{2}\right) d x<\epsilon,
\end{aligned}
$$

where $T^{a} \tilde{\rho}(x)=: \tilde{\rho}\left(x+a \mathbf{e}_{3}\right)$. 
The proof of Theorem 3.2 follows from several lemmas. The proofs of these lemmas are similar to those in [28], and therefore we only sketch them. First we have

Lemma 3.8. Suppose the angular momentum of the rotating star solutions satisfies (2.9), (3.1) and (3.2). For any $\rho(x) \in W_{M}$, if

$$
\lim _{r \rightarrow 0+} L\left(m_{\rho}(r)+m_{\tilde{\rho}}(r)\right) m_{\sigma}(r) r^{-2}=0,
$$

where $\sigma=\rho-\tilde{\rho}$, then

$$
d_{1}(\rho, \tilde{\rho}) \geq 0
$$

where $d_{1}$ is defined by (3.84).

Proof. For an axi-symmetric function $f(x)=f(r, z)\left(r=\sqrt{x_{1}^{2}+x_{2}^{2}}, z=x_{3}\right.$ for $x=$ $\left.\left(x_{1}, x_{2}, x_{3}\right)\right)$, we let

$$
\begin{gathered}
\hat{f}(r)=2 \pi r \int_{-\infty}^{+\infty} f(r, z) d z \\
m_{f}(r)=\int_{\left\{x: \sqrt{x_{1}^{2}+x_{2}^{2}} \leq r\right\}} f(x) d x=\int_{0}^{r} \hat{f}(s) d s,
\end{gathered}
$$

so that

$$
m_{f}^{\prime}(r)=\hat{f}(r)
$$

In order to show (3.91), we let

$$
\sigma(x)=(\rho-\tilde{\rho})(x)
$$

and for $0 \leq \alpha \leq 1$, we define

$$
\begin{aligned}
Q(\alpha) & =\frac{1}{2} \int \frac{(\tilde{\rho}+\alpha \sigma)(x) L\left(m_{\tilde{\rho}+\alpha \sigma}(r(x))\right)-\tilde{\rho}(x) L\left(m_{\tilde{\rho}}(r(x))\right)}{r^{2}(x)} d x \\
& -\alpha \iint_{r(x)}^{\infty} s^{-3} L\left(m_{\tilde{\rho}}(s)\right) d s \sigma(x) d x
\end{aligned}
$$

Then

$$
Q(0)=0, Q(1)=d_{1}(\rho, \tilde{\rho}) .
$$

By the assumption that $L^{\prime}(m) \geq 0$ for $0 \leq m \leq M$ (cf. (3.2)) and (3.90), we can show that

$$
Q^{\prime}(\alpha)=\int_{0}^{+\infty} \hat{\sigma}(r) \int_{r}^{\infty} s^{-3}\left(L\left(m_{\tilde{\rho}+\alpha \sigma}(s)\right)-L\left(m_{\tilde{\rho}}(s)\right)\right) d s d r
$$

and therefore

$$
Q(0)=Q^{\prime}(0)=0
$$

This is done by interchanging the order of integration and integrating by parts (details can be found in [28]). Differentiating (3.99) again and interchanging the order of integration, we get

$$
\frac{d^{2} Q(\alpha)}{d \alpha^{2}}=\alpha \int_{0}^{+\infty} s^{-3} L^{\prime}\left(m_{\tilde{\rho}+\alpha \sigma}(s)\right)\left(m_{\sigma}(s)\right)^{2} d s
$$


Therefore, if $L^{\prime}(m) \geq 0$ for $0 \leq m \leq M$, then

$$
\frac{d^{2} Q(\alpha)}{d \alpha^{2}} \geq 0, \text { for } 0 \leq \alpha \leq 1
$$

This, together with (3.99) and (3.97), yields $d_{1}(\rho, \tilde{\rho})=Q(1) \geq 0$.

Lemma 3.9. Let $(\rho, \mathbf{v})$ be a solution of the Cauchy problem (1.1), (3.52) as stated in Theorem 3.2, then

$$
\begin{aligned}
& E(\rho, \mathbf{v})(t)-F(\tilde{\rho}) \\
& =d(\rho(t), \tilde{\rho})+d_{1}(\rho(t), \tilde{\rho})-\frac{1}{8 \pi}\|\nabla B \rho(\cdot, t)-\nabla B \tilde{\rho}\|_{2}^{2} \\
& +\frac{1}{2} \int \rho\left(\left|v^{r}\right|^{2}+\left|v^{3}\right|^{2}\right)(x, t) d x .
\end{aligned}
$$

Proof. From A1)-A3), we can show

$$
j^{2}(x, t)=L\left(m_{\rho(t)}(r(x))\right), \quad x \in G_{t} .
$$

Therefore, by (3.68), we have

$$
\begin{aligned}
E(\rho(t), \mathbf{v}(t)) & =\int A(\rho)(x, t) d x+\frac{1}{2} \int \frac{\rho(x, t) L\left(m_{\rho(t)}(r(x))\right.}{r^{2}(x)} d x \\
& -\frac{1}{8 \pi} \int|\nabla B \rho|^{2}(x, t) d x+\frac{1}{2} \int \rho\left(\left|v^{r}\right|^{2}+\left|v^{3}\right|^{2}\right)(x, t) d x .
\end{aligned}
$$

(3.102) follows from (3.104) and the follow identities:

$$
\begin{aligned}
& \left(\|\nabla B \rho(\cdot, t)\|_{2}^{2}-\|\nabla B \tilde{\rho}\|_{2}^{2}\right) \\
& =\| \nabla(B \rho(\cdot, t))-\nabla B \tilde{\rho}) \|_{2}^{2}+2 \int \nabla B \tilde{\rho}(x) \cdot(\nabla B \rho(x, t)-\nabla B \tilde{\rho}(x)) d x \\
& =\| \nabla(B \rho(\cdot, t))-\nabla B \tilde{\rho}) \|_{2}^{2}-8 \pi \int B \tilde{\rho}(x)(\rho(x, t)-\tilde{\rho}(x)) d x .
\end{aligned}
$$

and

$$
\int \rho(x, t) d x=\int \tilde{\rho}(x) d x=M
$$

Having established these lemmas, the proof of Theorem 3.2 is the same as the proof of Theorem 3.1 in [28]. 


\section{Applications to White Dwarf and Supermassive Stars}

In this section, we want to verify the assumptions (3.4) and (3.5) in Theorem 3.1 for both white dwarfs and supermassive stars. Once we verify (3.4) and (3.5), we can apply Theorems 3.1 and 3.2. We begin with the following theorem which verifies (3.5) for white dwarfs, supermassive stars, and polytropes with $\gamma \geq 4 / 3$, in both the rotating and non-rotating cases.

Theorem 4.1. Assume that the pressure function $p$ satisfies (3.3). Then there exists a constant $\mathfrak{M}_{c}$ satisfying $0<\mathfrak{M}_{c}<\infty$ if $\gamma=4 / 3$ and $\mathfrak{M}_{c}=\infty$ if $\gamma>4 / 3$, such that if $M<\mathfrak{M}_{c}$, then (3.5) holds for $\rho \in W_{M}$.

Proof. Using (3.13), we have, for $\rho \in W_{M}$,

$$
\begin{aligned}
F(\rho) & =\int\left[A(\rho)+\frac{1}{2} \frac{\rho(x) L\left(m_{\rho}(r(x))\right)}{r(x)^{2}}-\frac{1}{2} \rho B \rho\right] d x \\
& \geq \int\left[A(\rho)+\frac{1}{2} \frac{\rho(x) L\left(m_{\rho}(r(x))\right)}{r(x)^{2}}\right] d x-C \int \rho^{4 / 3} d x\left(\int \rho d x\right)^{2 / 3} \\
& =\int\left[A(\rho)+\frac{1}{2} \frac{\rho(x) L\left(m_{\rho}(r(x))\right)}{r(x)^{2}}\right] d x-C M^{2 / 3} \int \rho^{4 / 3} d x
\end{aligned}
$$

Taking $p=1, q=4 / 3, r=\gamma$, and $a=\frac{\frac{3}{4} \gamma-1}{\gamma-1}$ (where $\gamma \geq 4 / 3$ is the constant in (3.3)) in Young's inequality (3.11), we obtain,

$$
\|\rho\|_{4 / 3} \leq\|\rho\|_{1}^{a}\|\rho\|_{\gamma}^{1-a}=M^{a}\|\rho\|_{\gamma}^{1-a}
$$

This, together with (3.16)-(3.18) yields

$$
\begin{aligned}
\int \rho^{4 / 3} d x & \leq M^{\frac{4}{3} a}\left(\int \rho^{\gamma} d x\right)^{b} \leq M^{\frac{4}{3} a}\left(\left(\rho^{*}\right)^{\gamma-1} M+\alpha \int A(\rho) d x\right)^{b} \\
& \leq C\left(M^{\frac{4}{3} a+b}\left(\rho^{*}\right)^{1 / 3}+\alpha M^{\frac{4}{3} a}\left(\int A(\rho) d x\right)^{b}\right)
\end{aligned}
$$

where $b=\frac{1}{3(\gamma-1)}, \alpha$ and $\rho^{*}$ are the constants in (3.17) and we have used the elementary inequality $(x+y)^{b} \leq C\left(x^{b}+y^{b}\right)$, for $x, y>0,0<b<1$, for some constant $C$. Therefore, (4.1) and (4.3) imply

$$
\int\left[A(\rho)+\frac{1}{2} \frac{\rho(x) L\left(m_{\rho}(r(x))\right)}{r(x)^{2}}\right] d x \leq F(\rho)+C \alpha M^{\frac{4}{3} a+\frac{2}{3}}\left(\int A(\rho) d x\right)^{b}+C M^{\frac{4}{3} a+b+\frac{2}{3}}\left(\rho^{*}\right)^{1 / 3} .
$$

If $\gamma>4 / 3$, then $0<b<1$, if $\gamma=4 / 3$, then $b=1$. Therefore (4.4) implies (3.5).

The next result shows that (3.4) holds for a wide class of (rotating or non-rotating) stars, including White Dwarfs. 
Theorem 4.2. Suppose that the pressure function p satisfies (3.3) and

$$
\lim _{\rho \rightarrow 0+} \frac{p(\rho)}{\rho^{\gamma_{1}}}=\beta,
$$

for some constants $\gamma_{1}>4 / 3$ and $0<\beta<+\infty$, and assume that the angular momentum (per unit mass) satisfies (2.9). Then there exists $\mathbb{M}_{c}$ satisfying $0<\mathbb{M}_{c}<+\infty$ if $\gamma=4 / 3$ and $\mathbb{M}_{c}=+\infty$ if $\gamma>4 / 3$ such that if $M<\mathbb{M}_{c}$, then (3.4) holds, where $\gamma$ is the constant in (3.3).

Remark 7. White dwarfs satisfy (3.3) and (4.5) with $\gamma=4 / 3$ and $\gamma_{1}=5 / 3$.

Proof of Theorem 4.2

Due to (3.3) and (4.5), we can apply Theorem 2.1. Let $\hat{\rho}(x) \in W_{M, S}$ be a minimizer $F(\rho)$ in $W_{M, S}$ as described in Theorem 2.1, and let

$$
G=\left\{x \in \mathbb{R}^{3}: \hat{\rho}(x)>0\right\} .
$$

Then $\bar{G}$ is a compact set in $\mathbb{R}^{3}$, and $\hat{\rho} \in C^{1}(G)$. Furthermore, there exists a constant $\mu<0$ such that

$$
\left\{\begin{array}{l}
A^{\prime}(\hat{\rho}(x))+\int_{r(x)}^{\infty} L\left(m_{\hat{\rho}}(s) s^{-3} d s-B \hat{\rho}(x)=\mu, \quad x \in G,\right. \\
\int_{r(x)}^{\infty} L\left(m_{\hat{\rho}}(s) s^{-3} d s-B \hat{\rho}(x) \geq \mu, \quad x \in \mathbb{R}^{3}-G .\right.
\end{array}\right.
$$

It follows from [2] that there exists $\hat{\rho} \in W_{M, S} \subset W_{M}$ such that $F(\hat{\rho})=\inf _{\rho \in W_{M, S}} F(\rho)$. It is easy to verify that the triple $(\hat{\rho}, \hat{\mathbf{v}}, \hat{\Phi})$ is a time-independent solution of the Euler-Poisson equations (1.1) in the region $G=\left\{x \in \mathbb{R}^{3}: \hat{\rho}(x)>0\right\}$, where $\hat{\mathbf{v}}=\left(-\frac{x_{2} J\left(m_{\hat{\rho}}(r)\right)}{r}, \frac{x_{1} J\left(m_{\hat{\rho}}(r)\right)}{r}, 0\right)$ and $\hat{\Phi}=-B \hat{\rho}$. Therefore

$$
\nabla_{x} p(\hat{\rho})=\hat{\rho} \nabla_{x}(B \hat{\rho})+\hat{\rho} L\left(m_{\hat{\rho}}\right) r(x)^{-3} \mathbf{e}_{r}, x \in G,
$$

where $\mathbf{e}_{r}=\left(\frac{x_{1}}{r(x)}, \frac{x_{2}}{r(x)}, 0\right)$. Moreover, it is proved in [4] that the boundary $\partial G$ of $G$ is smooth enough to apply the Gauss-Green formula on G. Applying the Gauss-Green formula on G and noting that $\left.\hat{\rho}\right|_{\partial G}=0$, we obtain,

$$
\int_{G} x \cdot \nabla_{x} p(\hat{\rho}) d x=-3 \int_{G} p(\hat{\rho}) d x=-3 \int p(\hat{\rho}) d x .
$$

As in [28], we have

$$
\int_{G} x \cdot \hat{\rho} \nabla_{x} B \hat{\rho} d x=-\frac{1}{2} \int_{G} \hat{\rho} B \hat{\rho} d x=-\frac{1}{2} \int \hat{\rho} B \hat{\rho} d x .
$$

Next, since $x \cdot \mathbf{e}_{r}=r(x)$, we have

$$
\begin{aligned}
& \int_{G} x \cdot \hat{\rho}(x) L\left(m_{\hat{\rho}}(r(x)) r^{-3}(x) \mathbf{e}_{r} d x\right. \\
& =\int_{G} \hat{\rho}(x) L\left(m_{\hat{\rho}}(r(x)) r^{-2}(x) d x\right. \\
& =\int \hat{\rho}(x) L\left(m_{\hat{\rho}}(r(x)) r^{-2}(x) d x .\right.
\end{aligned}
$$


Therefore, from (4.8)-(4.10) we have

$$
-3 \int p(\hat{\rho}) d x=-\frac{1}{2} \int \hat{\rho} B \hat{\rho} d x+\int \hat{\rho}(x) L\left(m_{\hat{\rho}}(r(x)) r^{-2}(x) d x .\right.
$$

Let $\bar{\rho}(x)=b^{3} \hat{\rho}(b x)$, for $b>0$; then $\bar{\rho} \in W_{M}$. Also, it is easy to verify that the following identities hold,

$$
\begin{gathered}
\int \bar{\rho} B \bar{\rho} d x=\int_{\mathbb{R}^{3}} \int_{\mathbb{R}^{3}} \frac{\bar{\rho}(x) \bar{\rho}(y)}{|x-y|} d x d y \\
=b \iint_{\mathbb{R}^{3}} \int_{\mathbb{R}^{3}} \frac{\hat{\rho}(x) \hat{\rho}(y)}{|x-y|} d x d y=b \int \hat{\rho} B \hat{\rho} d x \\
\int A(\bar{\rho}) d x=b^{-3} \int A\left(b^{3} \hat{\rho}(x)\right) d x .
\end{gathered}
$$

Moreover, for $r \geq 0$,

$$
\begin{aligned}
m_{\bar{\rho}}(r) & =2 \pi \int_{0}^{r} s \int_{-\infty}^{\infty} \bar{\rho}(s, z) d s d z \\
& =2 \pi \int_{0}^{r} s \int_{-\infty}^{\infty} \hat{\rho}(b s, b z) d s d z \\
& =2 \pi \int_{0}^{b r} s^{\prime} \int_{-\infty}^{\infty} \rho\left(s^{\prime}, z^{\prime}\right) d s^{\prime} d z^{\prime} \\
& =m_{\rho}(b r)
\end{aligned}
$$

Therefore,

$$
\begin{aligned}
\int \frac{\bar{\rho}(x) L\left(m_{\bar{\rho}}(r(x))\right)}{r(x)^{2}} d x & =\int \frac{b^{3} \hat{\rho}(x) L\left(m_{\hat{\rho}}(b r(x))\right)}{r(x)^{2}} d x \\
& =b^{2} \int \frac{\hat{\rho}(x) L\left(m_{\hat{\rho}}(r(x))\right)}{r(x)^{2}} d x .
\end{aligned}
$$

It follows from (4.12)-(4.15) that

$$
\begin{aligned}
F(\bar{\rho}) & =b^{-3} \int A\left(b^{3} \hat{\rho}\right) d x-\frac{1}{2} b \int \hat{\rho} B \hat{\rho} d x \\
& +\frac{b^{2}}{2} \int \frac{\hat{\rho}(x) L\left(m_{\hat{\rho}}(r(x))\right)}{r(x)^{2}} d x .
\end{aligned}
$$

Hence, (4.11) and (4.16) give

$$
\begin{aligned}
F(\bar{\rho}) & =\int\left(b^{-3} A\left(b^{3} \hat{\rho}\right)-3 b p(\hat{\rho}(x))\right) d x \\
& +\left(\frac{b^{2}}{2}-b\right) \int \frac{\hat{\rho}(x) L\left(m_{\hat{\rho}}(r(x))\right)}{r(x)^{2}} d x .
\end{aligned}
$$

In view of (2.9), we have

$$
\left(\frac{b^{2}}{2}-b\right) \int \frac{\hat{\rho}(x) L\left(m_{\hat{\rho}}(r(x))\right)}{r(x)^{2}} d x \leq 0
$$


if $b>0$ is small. It follows from $(3.9)$ that

$$
\frac{1}{2} \beta \rho^{\gamma_{1}} \leq p(\rho) \leq 2 \beta \rho^{\gamma_{1}}, \text { for small } \rho .
$$

Thus, when $b$ is small, since $\hat{\rho}$ is bounded, we have

$$
\frac{\beta}{2\left(\gamma_{1}-1\right)} b^{3 \gamma_{1}}(\hat{\rho})^{\gamma_{1}}(x) \leq A\left(b^{3} \hat{\rho}(x)\right) \leq \frac{2 \beta}{\gamma_{1}-1} b^{3 \gamma_{1}}(\hat{\rho})^{\gamma_{1}}(x),
$$

for $x \in \mathbb{R}^{3}$. Hence, (4.18) and (4.19) imply

$$
\begin{aligned}
& \int\left(b^{-3} A\left(b^{3} \hat{\rho}\right)-3 b p(\hat{\rho}(x))\right) d x \\
& \leq \beta \int\left(\frac{2}{\gamma_{1}-1} b^{3 \gamma_{1}-3}-\frac{3}{2}\right)(\hat{\rho})^{\gamma_{1}} d x .
\end{aligned}
$$

Since $\gamma_{1}>4 / 3$, we have $3 \gamma_{1}-3>1$. Therefore, we conclude that

$$
\int\left(b^{-3} A\left(b^{3} \hat{\rho}\right)-3 b p(\hat{\rho}(x))\right) d x<0,
$$

for small $b$. (3.4) follows from (4.17), (4.18) and (4.22). This completes the proof of Theorem 4.2.

We show next that if the angular momentum distribution is everywhere positive, we may apply the existence theorem of Friedman and Tarkington, [13, to conclude that (3.4) holds with no total mass restriction. This result applies also to White Dwarfs.

Theorem 4.3. Suppose that the pressure function $p$ satisfies (3.3) with $\gamma=4 / 3$ and (3.9) holds. Assume that the angular momentum (per unit mass) $J(m)=\sqrt{L(m)}$ satisfies (2.14), then 3.4) holds for $\rho \in W_{M}$ for any $0<M<+\infty$.

Proof. By the existence theorem in [13], if (2.14) is satisfied, then for any $0<M<+\infty$, there exits $\tilde{\rho} \in W_{M, S}$ such that $F(\tilde{\rho})=\inf _{\rho \in W_{M, S}} F(\rho)$. Also, all the properties of $\tilde{\rho}$ in Theorem 2.1 are satisfied. Moreover, the regularity of the boundary $\partial G$ is smooth enough to apply the Gauss-Green formula (cf. 4]). The proof now follows exactly as in Theorem 4.2.

We finally turn to the case of rotating supermassive stars.

Theorem 4.4. Consider suppermasive star; i.e.,

$$
p(\rho)=k \rho^{4 / 3}, \quad k>0 \text { is a constant. }
$$

If there exists $\hat{\rho} \in W_{M}$ such that $\hat{\rho} \in C^{1}(G) \cap C\left(\mathbb{R}^{3}\right)$ is a steady state solution of the EulerPoisson equation with the velocity field $\hat{\mathbf{v}}=\left(-\frac{x_{2} \sqrt{L}\left(m_{\hat{\rho}}(r)\right)}{r}, \frac{x_{1} \sqrt{L}\left(m_{\hat{\rho}}(r)\right)}{r}, 0\right)$ in an open bounded set $G \subset \mathbb{R}^{3}$ with the Lipschitz boundary $\partial G$, i.e.,

$$
\left\{\begin{array}{l}
\nabla_{x} p(\hat{\rho})=\hat{\rho} \nabla_{x}(B \hat{\rho})+\hat{\rho} L\left(m_{\hat{\rho}}\right) r(x)^{-3} \mathbf{e}_{r}, x \in G, \\
\hat{\rho}=0, \quad x \in \mathbb{R}^{3}-G .
\end{array}\right.
$$


then (3.4) holds provided L satisfies (2.9) and

$$
L\left(m_{0}\right)>0, \text { for some } m_{0} \in(0, M) .
$$

Proof. Following along the same lines as (4.7)-(4.10), we obtain the same equality as (4.11). Therefore,

$$
F(\hat{\rho})=-\frac{1}{2} \int \hat{\rho}(x) L\left(m_{\hat{\rho}}(r(x)) r^{-2}(x) d x,\right.
$$

in view of (4.23) and (4.11). Since $\hat{\rho} \in C^{1}(G) \cap C\left(\mathbb{R}^{3}\right)$ and $\hat{\rho}=0$ for $x \in \mathbb{R}^{3}-G$, it is easy to show that $m_{\hat{\rho}}(r)$ is continuous in $r$. Moreover, $m_{\hat{\rho}}(0)=0$ and $m_{\hat{\rho}}(R)=M$, where $R=\max _{x \in \bar{G}}\left(r(x)\right.$. Therefore, there exits $r_{0} \in(0, M)$ such that

$$
m_{\hat{\rho}}\left(r_{0}\right)=m_{0}
$$

where $m_{0}$ is the constant in (4.25). Thus,

$$
L\left(m_{\hat{\rho}}\left(r_{0}\right)\right)>0
$$

in view of (4.25). Since $m_{\hat{\rho}}(r)$ is continuous in $r$ and $L(m)$ is continuous in $m$, we conclude that

$$
\int \hat{\rho}(x) L\left(m_{\hat{\rho}}(r(x)) r^{-2}(x) d x>0 .\right.
$$

The inequality (3.4) now follows from (4.26)).

The preceding theorems, together with Theorem 3.2 show that polytropes $\left(p(\rho)=k \rho^{\gamma}\right)$ with $\gamma>4 / 3$ and White Dwarf stars, in both the rotating and non-rotating cases, as well as rotating Supermassive stars are dynamically stable. Moreover, if the angular momentum distribution is not everywhere positive and the pressure p behaves asymptotically near infinity like $\rho^{4 / 3}$, then dynamic stability holds only under a (Chandrasekhar) mass restriction, $M \leq$ $M_{c}$.

\section{$5 \quad$ Nonlinear Dynamical Stability of Non-Rotating White Dwarf Stars With General Perturbations}

The dynamical stability results in Section 3 apply for axi-symmetric perturbations. In this section, we prove the nonlinear dynamical stability for non-rotating white dwarf stars with general perturbations. For white dwarf stars, as mentioned before, the pressure function satisfies

$$
p \in C^{1}[0,+\infty), \lim _{\rho \rightarrow 0+} \frac{p(\rho)}{\rho^{\gamma_{1}}}=\beta, \lim _{\rho \rightarrow \infty} \frac{p(\rho)}{\rho^{\gamma}}=K, p^{\prime}(\rho)>0 \text { for } \rho>0
$$


where $\gamma_{1}>4 / 3,0<\beta<+\infty$ and $0<K<+\infty$ are constants. In this section, we always assume that the pressure function satisfies (5.1). First, we define for $0<M<+\infty$,

$$
\begin{gathered}
X_{M}=\left\{\rho: \mathbb{R}^{3} \rightarrow \mathbb{R}, \rho \geq 0, \text { a.e., } \int \rho(x) d x=M,\right. \\
\left.\int\left[A(\rho(x))+\frac{1}{2} \rho(x) B \rho(x)\right] d x<+\infty\right\},
\end{gathered}
$$

where $A(\rho)$ is the function given in (2.5). For $\rho \in X_{M}$, we define the energy functional $G$ for non-rotating stars by

$$
G(\rho)=\int\left[A(\rho(x))-\frac{1}{2} \rho(x) B \rho(x)\right] d x .
$$

We begin with the following theorem.

Theorem 5.1. Suppose that the pressure function $p$ satisfies (5.1). Let $\tilde{\rho}_{N}$ be a minimizer of the energy functional $G$ in $X_{M}$ and let

$$
\Gamma_{N}=\left\{x \in \mathbb{R}^{3}: \tilde{\rho}_{N}(x)>0\right\}
$$

then there exists a constant $\lambda_{N}$ such that

$$
\left\{\begin{array}{l}
A^{\prime}\left(\tilde{\rho}_{N}(x)\right)-B \tilde{\rho}_{N}(x)=\lambda_{N}, \quad x \in \Gamma_{N}, \\
-B \tilde{\rho}_{N}(x) \geq \lambda_{N}, \quad x \in \mathbb{R}^{3}-\Gamma_{N} .
\end{array}\right.
$$

The proof of this theorem is well-known, cf. [32] or [2].

Remark 8. 1) We call the minimizer $\tilde{\rho}_{N}$ of the functional $G$ in $X_{M}$ a non-rotating star solution.

2) It follows from [24] that the minimizer $\tilde{\rho}_{N}$ of the functional $G$ in $X_{M}$ is actually radial, and has a compact support.

Similar to Theorem 3.1, we have the following compactness theorem.

Theorem 5.2. Suppose that the pressure function $p$ satisfies (5.1). There exists a constant $M^{c}\left(0<M^{c}<\infty\right)$ such that if $M<M^{c}$, then the following hold:

$$
\inf _{\rho \in X_{M}} G(\rho)<0
$$

(2) for $\rho \in X_{M}$,

$$
\int A(\rho)(x) d x \leq C_{1} G(\rho)+C_{2},
$$

for some positive constants $C_{1}$ and $C_{2}$,

(3) if $\left\{\rho^{i}\right\} \subset X_{M}$ is a minimizing sequence for the functional $G$, then there exist a sequence of 
translations $\left\{x^{i}\right\} \subset \mathbb{R}^{3}$, a subsequence of $\left\{\rho^{i}\right\}$, (still labeled $\left\{\rho^{i}\right\}$ ), and a function $\tilde{\rho}_{N} \in X_{M}$, such that for any $\epsilon>0$ there exists $R>0$ with

$$
\int_{|x| \geq R} T \rho^{i}(x) d x \leq \epsilon, \quad i \in \mathbb{N}
$$

and

$$
T \rho^{i}(x) \rightarrow \tilde{\rho}_{N}, \text { weakly in } L^{4 / 3}\left(\mathbb{R}^{3}\right) \text {, as } i \rightarrow \infty,
$$

where $T \rho^{i}(x):=\rho^{i}\left(x+x^{i}\right)$.

Moreover

(4)

$$
\nabla B\left(T \rho^{i}\right) \rightarrow \nabla B\left(\tilde{\rho}_{N}\right) \text { strongly in } L^{2}\left(\mathbb{R}^{3}\right) \text {, as } i \rightarrow \infty
$$

and

(5) $\tilde{\rho}$ is a minimizer of $G$ in $X_{M}$.

Proof. First, the proofs of (1) and (2) are the same as Theorems 4.1 and 4.2 by taking $L=0$ (it is easy to check the axial symmetry is not used the the proof of Theorems 4.1 and 4.2 if $L=0$ ). Lemmas 3.4, 3.5 and 3.7 still hold by taking $\gamma=4 / 3$ and $L=0$, and replacing $W_{M}$ by $X_{M}, F$ by $G$ and $f_{M}$ by $\inf _{\rho \in X_{M}} G(\rho)$. Also, it is easy to check that (3.25)-(3.29) in the proof of Lemma 3.6 still hold by replacing $f_{M}$ by $\inf _{\rho \in X_{M}} G(\rho)$. Therefore, following the proof of Lemma 3.6, we conclude:

If $\left\{\rho^{i}\right\} \subset X_{M}$ is a minimizing sequence for $G$, then there exists constant $\delta_{0}>0, i_{0} \in \mathbf{N}$ and $x^{i} \in \mathbb{R}^{3}$, such that

$$
\int_{B_{1}\left(x^{i}\right)} \rho^{i}(x) d x \geq \delta_{0}, i \geq i_{0}
$$

Therefore, if we let

$$
T \rho^{i}(x):=\rho^{i}\left(x+x^{i}\right)
$$

then

$$
\int_{B_{1}(0)} T \rho^{i}(x) d x \geq \delta_{0}, i \geq i_{0}
$$

This is similar to (3.39). Having established this inequality and the other analogues of Lemmas 3.4, 3.5 and 3.7, we can prove this theorem in a similar manner as the proof of Theorem 3.1.

For the stability, we consider the Cauchy problem (1.1) with the initial data (3.53). We do not assume that the initial data have any symmetry.

Let $\tilde{\rho}_{N}$ be a minimizer of $G$ on $X_{M}$ and $\lambda_{N}$ be the constant in (5.5). For $\rho \in X_{M}$, we define

$$
\begin{aligned}
d\left(\rho, \tilde{\rho}_{N}\right) & =\int\left\{\left[A(\rho)-A\left(\tilde{\rho}_{N}\right)\right]-\left(\rho-\tilde{\rho}_{N}\right)\left(\lambda_{N}+B \tilde{\rho}_{N}\right\} d x\right. \\
& =\int\left\{\left[A(\rho)-A\left(\tilde{\rho}_{N}\right)\right]-B \tilde{\rho}_{N}\left(\rho-\tilde{\rho}_{N}\right)\right\} d x
\end{aligned}
$$


where we have used the identity

$$
\int \rho d x=\int \tilde{\rho}_{N} d x=M
$$

for $\rho \in X_{M}$. By a similar argument as (3.82) and (3.83), we have

$$
d\left(\rho, \tilde{\rho}_{N}\right) \geq 0
$$

for any $\rho \in X_{M}$, in view of (4.6). Our nonlinear stability theorem of non-rotating white dwarf star solutions is the following theorem, which extends the results in 32 .

Theorem 5.3. Suppose that the pressure function satisfies (5.1). Let $\tilde{\rho}_{N}$ be a minimizer of the functional $G$ in $X_{M}$, and assume that it is unique up to a translation $\rho_{N}(x) \rightarrow \rho_{N}(x+y)$. Let $(\rho, \mathbf{v}, \Phi)(x, t)$ be a weak solution of the Cauchy problem (1.1) and (3.52) satisfying

$$
\int \rho(x, t)=\int \rho_{0}(x)=\int \rho_{N}(x) d x=M
$$

If the total energy $E(t)$ (cf. (3.56)) is non-increasing with respect to $t$, then there exists a constant $M^{c}\left(0<M^{c}<\infty\right)$ such that if $M<M^{c}$, then for every $\epsilon>0$, there exists a number $\delta>0$ such that if

$$
d\left(\rho_{0}, \tilde{\rho}_{N}\right)+\frac{1}{8 \pi}\left\|\nabla B \rho_{0}-\nabla B \tilde{\rho}_{N}\right\|_{2}^{2}+\frac{1}{2} \int \rho_{0}(x)\left(\left|v_{0}\right|^{2}\right)(x) d x<\delta
$$

then there is a translation $y \in \mathbb{R}^{3}$ such that, for every $t>0$

$$
\left.d\left(\rho(t), T^{y} \tilde{\rho}_{N}\right)+\frac{1}{8 \pi}\left\|\nabla B \rho(t)-\nabla B T^{y} \tilde{\rho}_{N}\right\|_{2}^{2}+\frac{1}{2} \int \rho(x, t)|v(x, t)|^{2}\right) d x<\epsilon,
$$

where $T^{y} \tilde{\rho}_{N}(x)=: \tilde{\rho}_{N}(x+y)$.

The proof of this theorem follows from the compactness result (Theorem 5.2), and the arguments in [28] and [32], and is thus omitted.

\section{Acknowledgments}

Luo was supported in part by the National Science Foundation under Grants DMS-0606853 and DMS-0742834. Smoller was supported in part by the National Science Foundation under Grant DMS-0603754.

\section{References}

[1] L. Ambrosio, Transport equation and Cauchy problem for $B V$ vector fields. Invent. Math. 158, no. 2, 227-260 (2004).

[2] G. Auchmuty and R. Beals , Variational solutions of some nonlinear free boundary problems, Arch. Rat. Mech. Anal.43, 255-271 (1971). 
[3] G. Auchmuty, The global branching of rotating stars, Arch. Rat. Mech. Anal. 114, 179194 (1991).

[4] L. Caffarelli and A. Friedman, The shape of axi-symmetric rotating fluid, J. Funct. Anal., 694, 109-142 (1980).

[5] S. Chandrasekhar, Phil. Mag. 11, 592 (1931); Astrophys. J. 74, 81 (1931); Monthly Notices Roy. Astron. Soc. 91, 456 (1931); Rev. Mod. Phys. 56, 137 (1984).

[6] S. Chandrasekhar, Introduction to the Stellar Structure, University of Chicago Press (1939).

[7] Chanillo, Sagun and Li, Yan Yan, On diameters of uniformly rotating stars. Comm. Math. Phys. 166, no. 2, 417-430 (1994).

[8] G. Q. Chen \& H. Frid, Extended divergence-measure fields and the Euler equations for gas dynamics, Comm. Math. Phys. 236, no. 2, 251-280 (2003).

[9] R.J. Di Perna \& P. L. Lions, Ordinary differential equations, transport theory and Sobolev spaces. Invent. Math. 98, 511-547 (1989).

[10] Y. Deng, T.P. Liu, T. Yang \& Z.Yao, Solutions of Euler-Poisson equations for gaseous stars, Arch. Rat. Mech. Anal. 164 , no. 3, 261-285 (2002).

[11] R. H. Fowler, Monthly Notices Roy. Astron. Soc. 87, 114 (1926).

[12] A. Friedman \& B. Turkington, Asymptotic estimates for an axi-symmetric rotating fluid. J. Func. Anal. 37, 136-163 (1980).

[13] A. Friedman \& B. Turkington, Existence and dimensions of a rotating white dwarf. J. Diff. Eqns. 42, 414-437 (1981).

[14] J. Jang, Nonlinear instability in gravitational Euler-Poisson system for $\gamma=\frac{6}{5}$, to appear in Arch. Rat. Mech. Anal..

[15] Y. Guo \& G. Rein, Stable steady states in stellar dynamics, Arch. Rat. Mech. Anal. 147, 225-243 (1999).

[16] Y. Guo \& G. Rein, Stable models of elliptical galaxies, Mon. Not. R. Astron. Soc. 344, 1296-1306, (2002).

[17] D. Gilbarg \& N. Trudinger, Elliptic Partial Differentail Equations of Second Order (2nd ed.), Springer (1983).

[18] M. Humi, Steady states of self-gravitating incompressible fluid in two dimensions. J. Math. Phys. 47, no. 9, 093101, 10 pp (2006). 
[19] L, Landau, Phys. Z. Sowjetunion 1, 285 (1932)

[20] P. Lax, Shock Waves and Entropy, Contribution to Nonlinear Functional Analysis, E. A. Zarantonello, ed., Academic Press, New York 603-634 (1971).

[21] N. R. Lebovitz, The virial tensor and its application to self-gravitating fluids, Astrophys. J. 134 (1961) 500-536.

[22] N. R. Lebovitz \& A. Lifschitz, Short-wavelength instabilities of Riemann ellipsoids, Philos. Trans. Roy. Soc. London Ser. A 354, no. 1709, 927-950 (1996).

[23] Li, Yan Yan, On uniformly rotating stars, Arch. Rat. Mech. Anal. 115, no. 4, 367-393 (1991).

[24] E. H. Lieb \& H. T. Yau, The Chandrasekhar theory of stellar collapse as the limit of quantum mechanics. Comm. Math. Phys. 112, no. 1, 147-174 (1987).

[25] S. S. Lin, Stability of gaseous stars in spherically symmetric motions, SIAM J. Math. Anal. 28, no. 3, 539-569 (1997).

[26] P.L. Lions, The concentration-compactness principle in the calculus of variations. The locally compact case. Part I, Ann. Inst. H. Poincaré Anal. Non Linéaire, 1, 109-145 (1984).

[27] T. Luo \& J. Smoller, Rotating fluids with self-gravitation in bounded domains. Arch. Rat. Mech. Anal. 173, no. 3, 345-377 (2004).

[28] T. Luo \& J. Smoller, Existence and Nonlinear Stability of Rotating Star Solutions of the Compressible Euler-Poisson Equations, Preprint, http://arxiv.org/PS_cache/gr-qc/pdf/0703/0703033v1.pdf, (to appear in Arch. Rat. Mech. Anal.).

[29] T. Makino, Blowing up of the Euler-Poisson equation for the evolution of gaseous star, Transport Theory and Statistical Physics, 21, 615-624 (1992).

[30] M. Reed \& B.Simon, Methods of Modern Mathematical Physics II: Fourier Analysis, Self-Adjointness, Academic Press, NewYork (1975).

[31] G. Rein, Reduction and a concentration-compactness principle for energy-casimir functionals, SIAM J. Math. Anal. Vol 33, No. 4, 896-912 (2001).

[32] G. Rein, Non-linear stability of gaseous stars. Arch. Rat. Mech. Anal. 168, no. 2, 115-130 (2003). 
[33] S. H. Shapiro \& S. A. Teukolsky, Black Holes, White Dwarfs, and Neutron Stars, WILEYVCH, (2004)

[34] J. Smoller, Shock Waves and Reaction-Diffusion Equations, 2nd Ed.), Springer, Berlin, New York (1994).

[35] J. L. Tassoul, Theory of Rotating Stars, Princeton University Press, Princeton (1978).

[36] D. Wang, Global Solutions and Stability for Self-Gravitating Isentropic Gases, J. of Math. Anal. \& Appl., 229, 530-542 (1999).

[37] S. Weinberg, Gravitation and Cosmology John Wiley and Sons, New York, 1972.

Tao Luo

E-mail: tluo@wpi.edu

or

t148@georgetown.edu

Joel Smoller

E-mail: smoller@umich.edu 